Supporting Information for

\title{
A Cubic 3D Covalent Organic Framework with nbo Topology
}

Xue Wang, †‡ Mounib Bahri,§ Zhiwei Fu, Browning,§ Samantha Y. Chong, ${ }^{\ddagger}$ Linjiang Chen, ${ }^{* \dagger \neq ~ J o h n ~ W . ~ W a r d, ~}{ }^{*+\neq}$ and Andrew I. Cooper*†

† Leverhulme Research Centre for Functional Materials Design, University of Liverpool, Liverpool, L7 3NY, UK.

‡ Department of Chemistry and Materials Innovation Factory, University of Liverpool, Liverpool L69 7ZD, UK.

§ Albert Crewe Centre for Electron Microscopy, University of Liverpool, Liverpool, L69 3GL, UK. 
Table of Contents

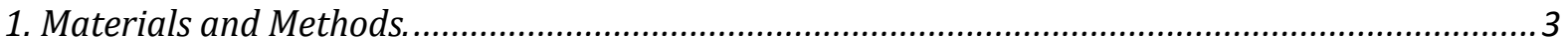

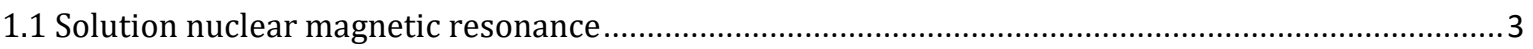

1.2 Solid-state ${ }^{13} \mathrm{C}$ CP MAS and ${ }^{11} \mathrm{~B}$ MAS nuclear magnetic resonance ................................................... 3

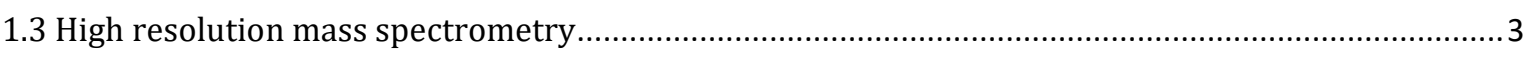

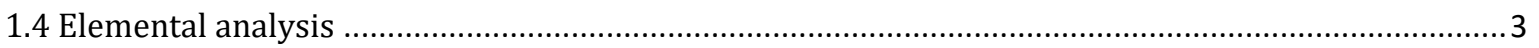

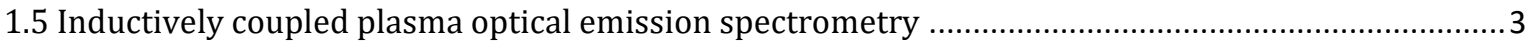

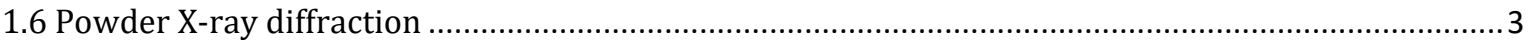

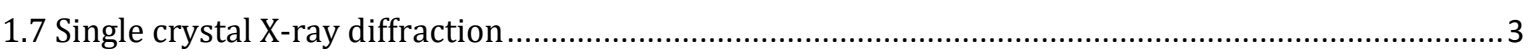

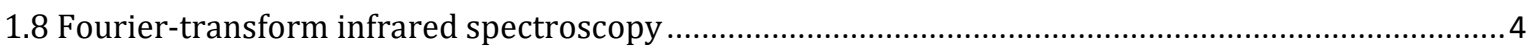

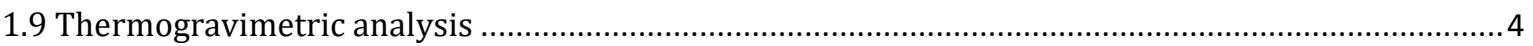

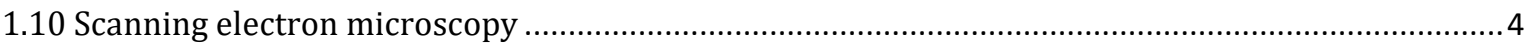

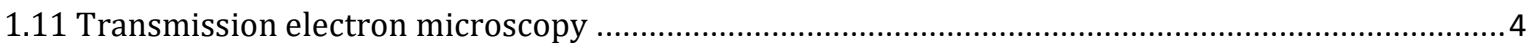

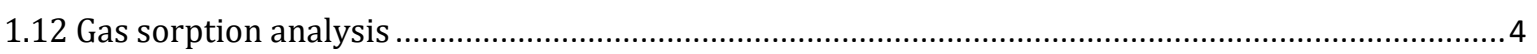

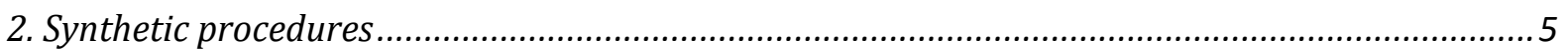

3. Solution NMR Spectra ..............................................................................................9

4. Conformations reported for spiroborate units in the Cambridge Structural Database (CSD) .....12

5. Single Crystal Structure of Model Compounds ................................................................. 12

6. Fourier-transform infrared spectroscopy .................................................................. 16

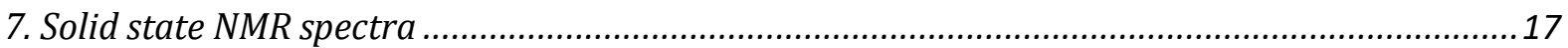

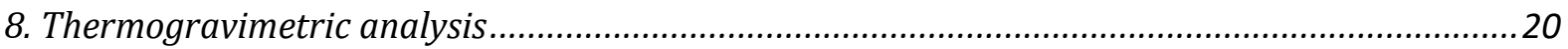

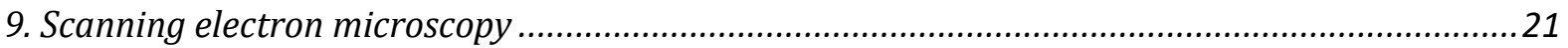

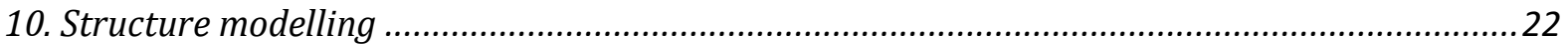

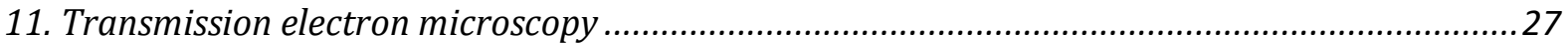

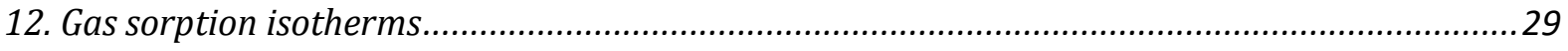

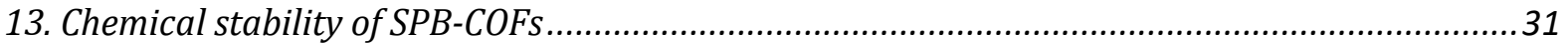

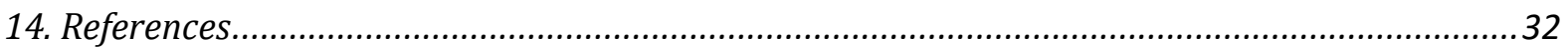




\section{Materials and Methods.}

All reagents were obtained from Sigma-Aldrich, Manchester Organics, or TCI Europe. Anhydrous solvents were purchased from Sigma-Aldrich, Acros Organics or Fisher Scientific. All chemicals were used without further purification. All gases for sorption analysis were supplied by BOC at a purity of $\geq 99.9 \%$. Reactions were carried out under nitrogen atmosphere using standard Schlenk techniques.

\subsection{Solution nuclear magnetic resonance}

NMR spectra were recorded on a Bruker Avance 400 NMR spectrometer, operating at frequencies of 400 $\mathrm{MHz}\left({ }^{1} \mathrm{H}\right)$ and $100 \mathrm{MHz}\left({ }^{13} \mathrm{C}\right)$ and referenced against the residual ${ }^{1} \mathrm{H}$ or ${ }^{13} \mathrm{C}$ signal of the solvent. ${ }^{11} \mathrm{~B}$ spectra operating at $128 \mathrm{MHz}$ using deuterium lock for referencing.

\subsection{Solid-state ${ }^{13} \mathrm{C}$ CP MAS and ${ }^{11 B}$ MAS nuclear magnetic resonance}

Solid-state NMR experiments were performed on a Bruker Avance III HD spectrometer using the Durham University (UK) solid-state NMR service. Carbon-13 magic-angle spinning measurements were carried out at $100.63 \mathrm{MHz}$ using a Bruker Avance III HD spectrometer and $4 \mathrm{~mm}$ (rotor o.d.) probe. Spectra were acquired at a spin rate of $10 \mathrm{kHz}$. Cross-polarisation (CP) spectra were recorded with TOSS spinning sideband suppression, $0.8 \mathrm{~ms}$ contact time and with a recycle delay of $1 \mathrm{~s}$. Carbon spectral referencing is relative to neat tetramethylsilane, carried out by setting the high-frequency signal from an external sample of adamantane to $38.5 \mathrm{ppm}$. $50 \mathrm{~Hz}$ of line broadening was added to improve the signal to noise. Boron-11 magic-angle spinning measurements were carried out at $128.39 \mathrm{MHz}$ using a Bruker Avance III HD spectrometer and $4 \mathrm{~mm}$ (rotor o.d.) probe. Spectra were acquired at a spin rate of $20 \mathrm{kHz}$. All direct excitation 11B spectra were acquired with a 1 us 30-degree solid pulse which was determined from a 6 us solution pulse determined on $\mathrm{BF}_{3} / \mathrm{OEt}_{2}$. The spectra were acquired with a recycle delay of $1 \mathrm{~s}$ determined on the sample. Boron spectral referencing is relative to $\mathrm{BF}_{3} / \mathrm{OEt}_{2}$. Since the probe used has a boron background, a spectrum of an empty rotor was collected, and this was subtracted from all spectra.

\subsection{High resolution mass spectrometry}

High resolution mass spectrometry (HR-MS) as performed on an Agilent Technologies 6530B accuratemass QTOF mixed ESI/APCI mass spectrometer (capillary voltage $4000 \mathrm{~V}$, fragmentor $225 \mathrm{~V}$ ) in positiveion detection mode.

\subsection{Elemental analysis}

CHN analysis was performed on a Thermo EA1112 Flash CHNS-0 Analyzer using standard microanalytical procedures.

\subsection{Inductively coupled plasma optical emission spectrometry}

Inductively coupled plasma optical emission spectrometry (ICP-OES) measurements were conducted on an ICP-OES Agilent 5110. Samples were digested in concentrated nitric acid (67-69\%, trace metal analysis grade) by microwave, and then diluted using distilled water.

\subsection{Powder X-ray diffraction}

Laboratory powder X-ray diffraction (PXRD) data patterns were collected in transmission mode on samples held on thin Mylar film in aluminium well plates on a Panalytical Empyrean diffractometer equipped with a high throughput screening (HTS) XYZ stage, X-ray focusing mirror, and PIXcel detector, using $\mathrm{Cu}-\mathrm{K} \alpha$ radiation. For HT screening, PXRD patterns were measured over the $2 \theta$ range $1-56^{\circ}$ in $0.013^{\circ}$ steps over 30 minutes.

\subsection{Single crystal X-ray diffraction}

Single crystal X-ray data for the model compounds were measured on a Rigaku MicroMax-007 HF rotating anode diffractometer (Mo-K $\alpha$ radiation, $\lambda=0.71073 \AA$, Kappa 4-circle goniometer, Rigaku Saturn724+ detector), and data reduction was performed using CrysAlisPro. Structures were solved with SHELXT ${ }^{1}$ and refined by full-matrix least squares on $|F|^{2}$ by SHELXL, ${ }^{2}$ interfaced through the programme OLEX2. ${ }^{3}$ All non$\mathrm{H}$ atoms were refined anisotropically. $\mathrm{H}$ atoms bonded to $\mathrm{C}$ atoms were fixed in geometrically estimated 
positions and refined using the riding model. $\mathrm{H}$ atoms bonded to $\mathrm{N}$ atoms were located in the different maps and refined isotropically. For full refinement details, see Tables S2 and Table S3.

\subsection{Fourier-transform infrared spectroscopy}

Transmission FT-IR spectra were obtained with an attenuated total reflectance (ATR) method on a Bruker Tensor-27 spectrometer at room temperature.

\subsection{Thermogravimetric analysis}

TGA analysis was carried out using a TA Q5000IR analyzer with an automated vertical overhead thermobalance. Samples were heated at a rate of $10^{\circ} \mathrm{C} / \mathrm{min}$ under a dry nitrogen gas flow.

\subsection{Scanning electron microscopy}

SEM images were recorded using a Hitachi S-4800 cold field emission scanning electron microscope (FESEM). Samples were prepared by depositing the dry powders on a silicon disk and then coating the samples with Chromium using an Emitech K550X automated sputter coater.

\subsection{Transmission electron microscopy}

TEM images were obtained using a JEOL 2100+ microscope operating at 200KV equipped with a Gatan Rio Camera. Since COF materials are electron beam sensitive, the electron beam damage to the specimen was minimized as much as possible. A single HR-TEM image was recorded with an exposure time of 2 seconds. After drift compensation, some frames can be superimposed to increase the signal-to-noise (SN) ratio.

SPB-COF crystals were dispersed in anhydrous acetone by ultrasonication and drop-cast on a holey carbon film on 200 mesh copper grids.

\subsection{Gas sorption analysis}

Surface areas were measured by nitrogen sorption at 77.3 K. Powder samples were degassed offline at room temperature, followed by degassing on the analysis port under vacuum at room temperature for 12 hours. Isotherms were measured using a Micromeritics 2420 volumetric adsorption analyzer. Surface areas were calculated in the relative pressure $\left(P / P_{0}\right)$ range from 0.05 to 0.30 of the adsorption branch. 


\section{Synthetic procedures}

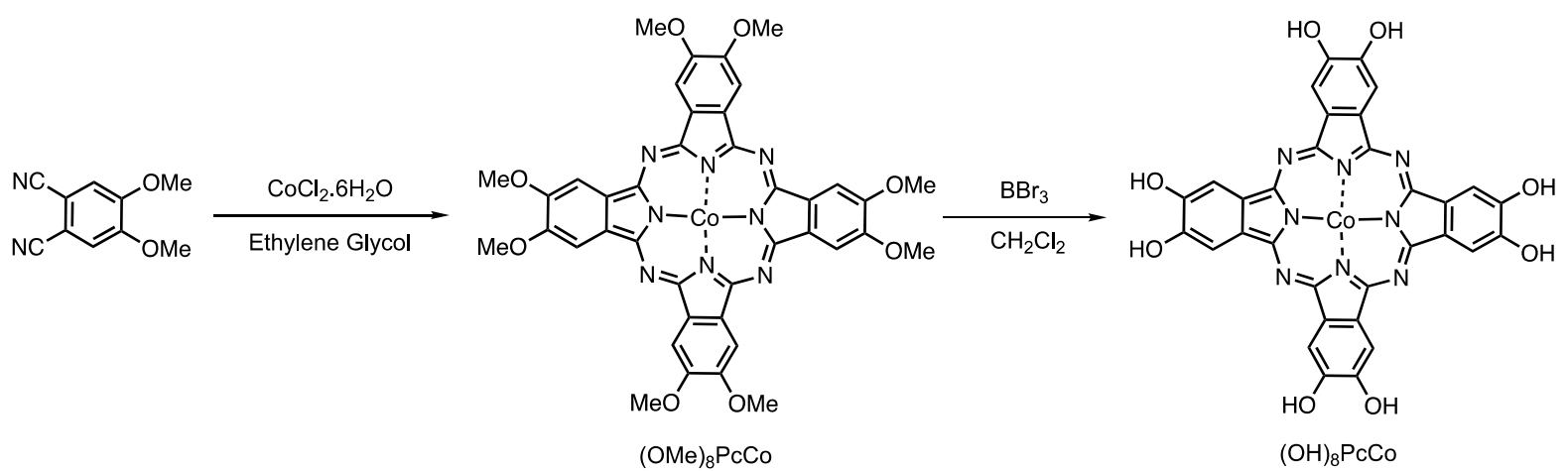

Scheme S1. Synthesis of $(\mathrm{OH})_{8} \mathrm{PcC}$.

$(\mathrm{OH})_{8} \mathrm{PcC}$ o was synthesized according to a reported procedure with some modifications. ${ }^{4,5}$

Synthesis of (Me0) ${ }_{8} P c C o:$ Under $\mathrm{N}_{2}$ atmosphere, 4,5-dimethoxyphthalodinitrile $(1.129 \mathrm{~g}, 6.0 \mathrm{mmol})$ and cobalt (II) chloride hexahydrate $(0.238 \mathrm{~g}, 1.0 \mathrm{mmol})$ were heated in $20 \mathrm{~mL}$ of ethylene glycol at $200{ }^{\circ} \mathrm{C}$ for 5 hours, after which the mixture was cooled to about $100^{\circ} \mathrm{C}$ and an equal volume of water was added. The mixture was suction filtered while still hot using a Whatman PTFE membrane filter with pore size of 0.45 $\mu \mathrm{m}$, and the filter cake washed with hot water $(50 \mathrm{~mL})$. The crude product was suspended in $1.0 \mathrm{M} \mathrm{HCl}$ (aq.) $(100 \mathrm{~mL})$ and heated to $120{ }^{\circ} \mathrm{C}$ for 1 hours, then filtered, and washed with hot water $(50 \mathrm{~mL})$. Then neutralised by suspending in $1.0 \mathrm{M} \mathrm{NaOH}$ (aq.) $\left(100 \mathrm{~mL}\right.$ ) heated to $120^{\circ} \mathrm{C}$ for 1 hours, filtered, and washed with hot water until the filtrate became neutral. The solid material was washed with methanol and dried in vacuo at $60^{\circ} \mathrm{C}$ for 6 hours, and isolated in $41 \%$ yield as a black powder. MALDI-TOF MS for $\mathrm{C}_{40} \mathrm{H}_{32} \mathrm{CoN}_{8} \mathrm{O}_{8}$ (calcd. 811.17), found $\mathrm{m} / \mathrm{z}=811.04([\mathrm{M}]+$ ).

Synthesis of $(\mathbf{O H})_{8} P$ PcCo: Under $\mathrm{N}_{2}$ atmosphere, $(\mathrm{MeO})_{8} \mathrm{PcCo}(0.5 \mathrm{~g}, 0.62 \mathrm{mmol})$ was suspended in anhydrous dichloromethane $(40 \mathrm{~mL})$ and stirred at around $-5{ }^{\circ} \mathrm{C}$ in an ice bath for 10 minutes. Boron tribromide $\left(\mathrm{BBr}_{3}\right)$ solution $(1.0 \mathrm{M}$ in methylene chloride) $(24.7 \mathrm{~mL}, 24.70 \mathrm{mmol})$ was then added slowly under ice bath. Upon the complete addition of $\mathrm{BBr}_{3}$ solution, the mixture was warmed to room temperature and stirred for 3 days. The reaction mixture was then quenched under an ice bath with methanol $(20 \mathrm{~mL})$ was added slowly (dropwise at the beginning). The solvent was removed in vacuo and the residue washed with methanol $(30 \mathrm{~mL})$ and centrifuged. After repeating the cycle of washing and centrifuging more than 3 times, the precipitate was collected and dried in vacuum, to give $(\mathrm{OH})_{8} \mathrm{PcCo}$ in $70 \%$ yield as a black powder. MALDI-TOF MS for $\mathrm{C}_{32} \mathrm{H}_{16} \mathrm{CoN}_{8} \mathrm{O}_{8}$ (Calc. 699.04), found $\mathrm{m} / \mathrm{z}=699.24$ ([M]+). 


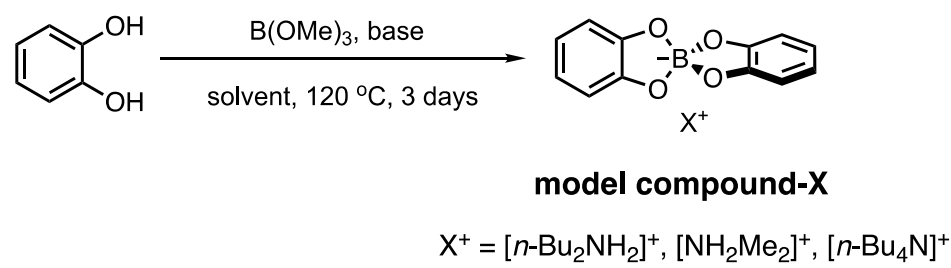

Scheme S2. Synthesis of spiroborate model compounds.

Synthesis of model compound-DBA (DBA = $\left.\left[\boldsymbol{n}-\mathbf{B u}_{2} \mathbf{N H}_{2}\right]^{+}\right)$: Under $\mathrm{N}_{2}$ atmosphere, 1,2-dihydroxybenzene $(1.05 \mathrm{~g}, 9.54 \mathrm{mmol})$ and $\mathrm{B}(\mathrm{OMe})_{3}(0.53 \mathrm{~mL}, 4.77 \mathrm{mmol})$ was dissolved in $N, N$-dibutylformamide (DBF) (50 $\mathrm{mL}$ ). The solution was then heated at $120^{\circ} \mathrm{C}$ for 3 days. During heating, the reaction changed from a transparent bright yellow solution to an opaque dark brown solution. After the reaction mixture was cooled to room temperature, the solvent was removed using vacuum distillation. The brown oil residue was then dissolved in very small amount of dichloromethane and then added dropwise to stirred cold diethyl ether $(300 \mathrm{~mL})$ to precipitate the product. The solid product was collected by filtration and dried in a vacuum oven at $80^{\circ} \mathrm{C}$ for 6 hours to give the desired product in $79 \%$ yield ( $1.35 \mathrm{~g}$ ) as a light grey crystalline powder. Single crystals of the model compound-DBA suitable for X-ray diffraction were grown from saturated ethyl acetate solution at room temperature for one week.

${ }^{1} \mathrm{H}$ NMR (400 MHz, DMSO- $\left.d_{6}\right): \delta=8.06(\mathrm{~s}, 2 \mathrm{H}), 6.48(\mathrm{~s}, 8 \mathrm{H}), 2.86(\mathrm{~m}, 4 \mathrm{H}), 1.53(\mathrm{dt}, 4 \mathrm{H}), 1.31(\mathrm{~m}, 4 \mathrm{H}), 0.89$ $(\mathrm{t}, J=8.0 \mathrm{~Hz}, 6 \mathrm{H}) \mathrm{ppm} .{ }^{13} \mathrm{C}$ NMR $\left(100 \mathrm{MHz}, \mathrm{DMSO}-d_{6}\right): \delta=151.54,117.29,107.60,46.54,27.56,19.22,13.47$ ppm. MS (ESI-) m/z calcd for $\mathrm{C}_{12} \mathrm{H}_{8} \mathrm{BO}_{4}$ [M]-: 227.05. Found: 227.0526. MS (ESI+) m/z calcd for $n-\mathrm{Bu}_{2} \mathrm{NH}_{2}$ [M]+: 130.16. Found: 130.1588. Anal. Cald for $\mathrm{C}_{20} \mathrm{H}_{28} \mathrm{BNO}_{4}$ : C: 67.19, H: 7.92, N: 3.92. Found: C: 67.07, H: 7.94, N: 3.71 .

Synthesis of model compound-DMA (DMA $=\left[\mathrm{NH}_{2} \mathrm{Me}_{2}\right]^{+}$): Following the same procedure as model compound-DBA above, but with $\mathrm{NHMe}_{2}(2.4 \mathrm{~mL}, 4.77 \mathrm{mmol}$, use $2.0 \mathrm{M}$ in THF) as the base and 1-methyl-2pyrrolidinone (NMP) as the solvent. During heating, the reaction changed from a transparent light-yellow solution to an opaque dark red solution. The product was obtained in $81 \%$ yield (1.05 g) as a brown powder. No single crystal structure was obtained for this model compound.

${ }^{1} \mathrm{H}$ NMR (400 MHz, DMSO-d6): $\delta=8.14(\mathrm{~s}, 2 \mathrm{H}), 6.47(\mathrm{~m}, 8 \mathrm{H}), 2.54(\mathrm{~s}, 6 \mathrm{H}) \mathrm{ppm} .{ }^{13} \mathrm{C}$ NMR (100 MHz, DMSO$\left.d_{6}\right): \delta=151.54,117.28,107.59,34.38$ ppm. MS (ESI-) m/z calcd for $\mathrm{C}_{12} \mathrm{H}_{8} \mathrm{BO}_{4}$ [M]-: 227.05. Found: 227.0526. MS (ESI+) m/z calcd for $\mathrm{NH}_{2} \mathrm{Me}_{2}$ [M]+: 46.07. Found: 46.0657. Anal. Cald for $\mathrm{C}_{14} \mathrm{H}_{16} \mathrm{BNO}_{4}: \mathrm{C}: 61.51, \mathrm{H}: 5.92$, N: 5.13. Found: C: 60.61, H: 5.91, N: 5.18.

Synthesis of model compound-TBA $\left(\left[n-\mathrm{Bu}_{4} \mathbf{N}\right]^{+}\right)$: Following the same procedure as model compoundDBA above, but with $n$ - $\mathrm{Bu} 4 \mathrm{NOH}_{3} 30 \mathrm{H}_{2} \mathrm{O}$ (3.81 g, $4.77 \mathrm{mmol}$ ) as the base and 1-methyl-2-pyrrolidinone (NMP) as the solvent. During heating, the reaction changed from a transparent brown-yellow solution to an opaque dark brown solution. The product was obtained in $80 \%$ yield ( $1.80 \mathrm{~g})$ as a brown powder. Single crystals of the model compound-TBA suitable for X-ray diffraction were grown by slow diffusion of diethyl ether into its saturated ethyl acetate or ethanol solution at room temperature for one week.

${ }^{1} \mathrm{H}$ NMR (400 MHz, DMSO- $\left.d_{6}\right): \delta=6.46(\mathrm{~m}, 8 \mathrm{H}), 3.15(\mathrm{~m}, 8 \mathrm{H}), 1.56(\mathrm{~m}, 8 \mathrm{H}), 1.30(\mathrm{~m}, 8 \mathrm{H}), 0.93(\mathrm{t}, J=8.0 \mathrm{~Hz}$, $12 \mathrm{H}) \mathrm{ppm} .{ }^{13} \mathrm{C}$ NMR $\left(100 \mathrm{MHz}\right.$, DMSO- $\left.d_{6}\right): \delta=151.56,117.25,107.57,57.49,23.04,19.19,13.47 \mathrm{ppm}$. MS (ESI-) m/z calcd for $\mathrm{C}_{12} \mathrm{H}_{8} \mathrm{BO}_{4}$ [M]-: 227.05. Found: 227.0491. MS (ESI+) m/z calcd for nBu4N [M]+: 242.28. Found: 242.2841. Anal. Cald for $\mathrm{C}_{28} \mathrm{H}_{44} \mathrm{BNO}_{4}$ : C: 71.59, H: 9.47, N: 2.98. Found: C: 70.82, H: 9.53, N: 3.24. 


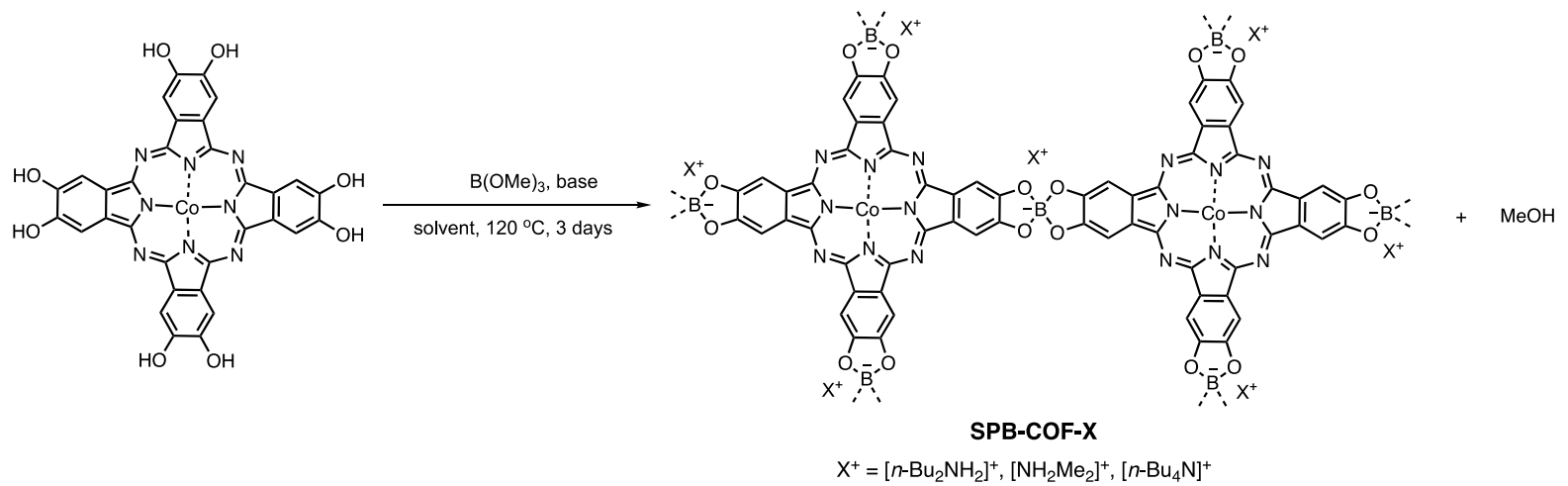

Scheme S3. Synthesis of SPB-COFs.

Synthesis of SPB-COF-DBA (DBA $\left.=\left[\boldsymbol{n}-\mathrm{Bu}_{2} \mathbf{N H}_{2}\right]^{+}\right)$: A $10 \mathrm{~mL}$ Pyrex tube was charged with $(\mathrm{OH})_{8}$ PcCo $(21.0$ $\mathrm{mg}, 0.03 \mathrm{mmol}), \mathrm{B}(\mathrm{OMe})_{3}(7.0 \mu \mathrm{L}, 0.06 \mathrm{mmol})$ and $N, N$-dibutylformamide (DBF) $(1.0 \mathrm{~mL})$. The mixture was sonicated at room temperature for 2 minutes, then flash frozen in a liquid $\mathrm{N}_{2}$ bath and degassed through three freeze-pump-thaw cycles and sealed under vacuum using a Schlenk line and oil pump. Upon warming to room temperature, the tube was put into a $120^{\circ} \mathrm{C}$ oven for 72 hours and then taken out of the oven, which produced a black, gel-like product. After cooling to room temperature, the product was washed with anhydrous acetone to give a dark powder. The obtained powder was immersed in anhydrous acetone, and the solvent was exchanged with fresh acetone three times with intervals of 1 hour. The wet sample was then transferred to a Critical Point Drier (Quorum-E3100AG); the sample was then washed and exchanged with liquid $\mathrm{CO}_{2}$ for 3-4 times with the interval of 1.5 hours, until all acetone in the material was successfully exchanged. After the final exchange, the system was heated to reach the critical point and the supercritical $\mathrm{CO}_{2}$ was then released slowly over 1 hour. After the chamber pressure returned to ambient, samples were then transferred into a gas adsorption test tube inside the glove box and degassed at room temperature for $12 \mathrm{~h}$ affording SPB-COF-DBA in 58\% yield $(17 \mathrm{mg})$ as a black powder. This sample was directly used for gas adsorption (several batches were combined together for $\mathrm{N}_{2}$ sorption). This batch of SPB-COF-DBA was used in all the experiments/measurements unless otherwise specified. Anal. Cald for $\left(\mathrm{C}_{8} \mathrm{H}_{2} \mathrm{Co}_{0.25} \mathrm{~N}_{2} \mathrm{O}_{2} \mathrm{~B}_{0.5}+\right.$ $\mathrm{C}_{4} \mathrm{H}_{10} \mathrm{~N}_{0.5}=\mathrm{C}_{48} \mathrm{H}_{48} \mathrm{CoN}_{10} \mathrm{O}_{8} \mathrm{~B}_{2}$ ): C: 59.22; H: 4.97; N: 14.39. Found: C: 50.97; H: 4.15; N: 12.22. ICP-OES analysis shows a Co content of $5.09 \mathrm{wt} . \%$.

Synthesis of SPB-COF-DMA (DMA $=\left[\mathrm{NH}_{2} \mathrm{Me}_{2}\right]^{+}$): Following the same procedure as SPB-COF-DBA above, but with $\mathrm{NHMe}_{2}(30.0 \mu \mathrm{L}, 0.06 \mathrm{mmol}, 2.0 \mathrm{M}$ in THF) as base and anhydrous 1-Methyl-2-pyrrolidinone (NMP) $(1.0 \mathrm{~mL})$ as the solvent. SPB-COF-DMA was obtained in $71 \%$ yield $(17.2 \mathrm{mg})$ as a black powder. This batch of SPB-COF-DMA was used in all the experiments/measurements unless otherwise specified. Anal. Cald for $\left(\mathrm{C}_{8} \mathrm{H}_{2} \mathrm{Co}_{0.25} \mathrm{~N}_{2} \mathrm{O}_{2} \mathrm{~B}_{0.5}+\mathrm{CH}_{4} \mathrm{~N}_{0.5}=\mathrm{C}_{36} \mathrm{H}_{24} \mathrm{CoN}_{10} \mathrm{O}_{8} \mathrm{~B}_{2}\right)$ : C: $53.70 ; \mathrm{H}: 3.00 ; \mathrm{N}: 17.40$. Found: $\mathrm{C}: 47.26 ; \mathrm{H}: 3.69$; $\mathrm{N}: 13.19$. ICP-OES analysis shows a Co content of $5.21 \mathrm{wt} . \%$.

Synthesis of SPB-COF-TBA (TBA $\left.=\left[\boldsymbol{n}-\mathrm{Bu}_{4} \mathbf{N}\right]^{+}\right)$: Following the same procedure as SPB-COF-DBA above, but with $n$ - $\mathrm{Bu}_{4} \mathrm{NOH} .30 \mathrm{H}_{2} \mathrm{O}(48.0 \mathrm{mg}, 0.06 \mathrm{mmol})$ as base and anhydrous 1-methyl-2-pyrrolidinone (NMP) $(1.0 \mathrm{~mL})$ as the solvent. SPB-COF-TBA was obtained in $83 \%$ yield $(30 \mathrm{mg})$ as a black powder. This batch of SPB-COF-TBA was used in all the experiments/measurements unless otherwise specified. Anal. Cald for $\left(\mathrm{C}_{8} \mathrm{H}_{2} \mathrm{Co}_{2.25} \mathrm{~N}_{2} \mathrm{O}_{2} \mathrm{~B}_{0.5}+\mathrm{C}_{8} \mathrm{H}_{18} \mathrm{~N}_{0.5}=\mathrm{C}_{64} \mathrm{H}_{80} \mathrm{CoN}_{10} \mathrm{O}_{8} \mathrm{~B}_{2}\right): \mathrm{C}: 64.17 ; \mathrm{H}: 6.73 ; \mathrm{N}: 11.69$. Found: C: $54.74 ; \mathrm{H}: 5.64 ;$ $\mathrm{N}: 10.98$. ICP-OES analysis shows a Co content of $4.54 \mathrm{wt} . \%$. 
Table S1. Synthetic conditions screened for the synthesis of SPB-COFs.

\begin{tabular}{|c|c|c|c|c|c|c|}
\hline Entry & $\begin{array}{c}\text { Solvent } \\
1\end{array}$ & Solvent 2 & Base & $\begin{array}{c}\text { Temperature } \\
\left({ }^{\circ} \mathrm{C}\right)\end{array}$ & $\begin{array}{l}\text { Time } \\
\text { (days) }\end{array}$ & Crystallinity \\
\hline 1 & DMF & - & - & 150 & 5 & Low \\
\hline 2 & DMF & - & $\mathrm{LiOH}$ & 120 & 3 & amorphous \\
\hline 3 & DMF & - & $\mathrm{LiOH}$ & 120 & 7 & Low \\
\hline 4 & $\begin{array}{l}\text { DMF } \\
(0.5)\end{array}$ & $\begin{array}{c}\text { Mesitylene } \\
(0.5)\end{array}$ & $\mathrm{LiOH}$ & 120 & 3 & Low \\
\hline 5 & $\begin{array}{l}\text { DMF } \\
(0.5)\end{array}$ & $\begin{array}{c}\text { Mesitylene } \\
(0.5)\end{array}$ & $\begin{array}{c}\mathrm{NHMe}_{2} \\
(2.0 \mathrm{M} \text { in THF) }\end{array}$ & 120 & 3 & Low \\
\hline 6 & DMAc & - & $\mathrm{LiOH}$ & 120 & 7 & amorphous \\
\hline 7 & DMAc & - & $\begin{array}{c}\mathrm{NHMe}_{2} \\
(2.0 \mathrm{M} \text { in THF })\end{array}$ & 120 & 7 & amorphous \\
\hline 8 & DEF & - & $\mathrm{LiOH}$ & 120 & 3 & amorphous \\
\hline 9 & DBF & - & $\mathrm{LiOH}$ & 120 & 3 & high \\
\hline 10 & $\mathrm{DBF}$ & - & pyridine & 120 & 3 & amorphous \\
\hline 11 & DBF & - & - & 120 & 3 & high \\
\hline 12 & NMP & - & $\mathrm{LiOH}$ & 120 & 3 & high \\
\hline 13 & NMP & - & $\begin{array}{c}\mathrm{NHMe}_{2} \\
(2.0 \mathrm{M} \text { in THF })\end{array}$ & 120 & 3 & high \\
\hline 14 & NMP & - & $\begin{array}{c}n-\mathrm{Bu}_{4} \mathrm{NOH} \\
(10 \% \text { in } \mathrm{MeOH})\end{array}$ & 120 & 3 & amorphous \\
\hline 15 & NMP & - & $n-\mathrm{Bu}_{4} \mathrm{NOH} \cdot 3 \mathrm{HH}_{2} \mathrm{O}$ & 120 & 3 & high \\
\hline 16 & NMP & - & pyridine & 120 & 3 & Low \\
\hline 17 & NMP & - & - & 120 & 3 & high \\
\hline
\end{tabular}

* All these synthetic conditions gave product with good to high yields. While we could also obtain crystalline product from synthesis entries 9,12 and 17, we did not follow these routes in more detail because it was difficult to model the counter-cation (we suspect a mixture of counter-cations). 


\section{Solution NMR Spectra}
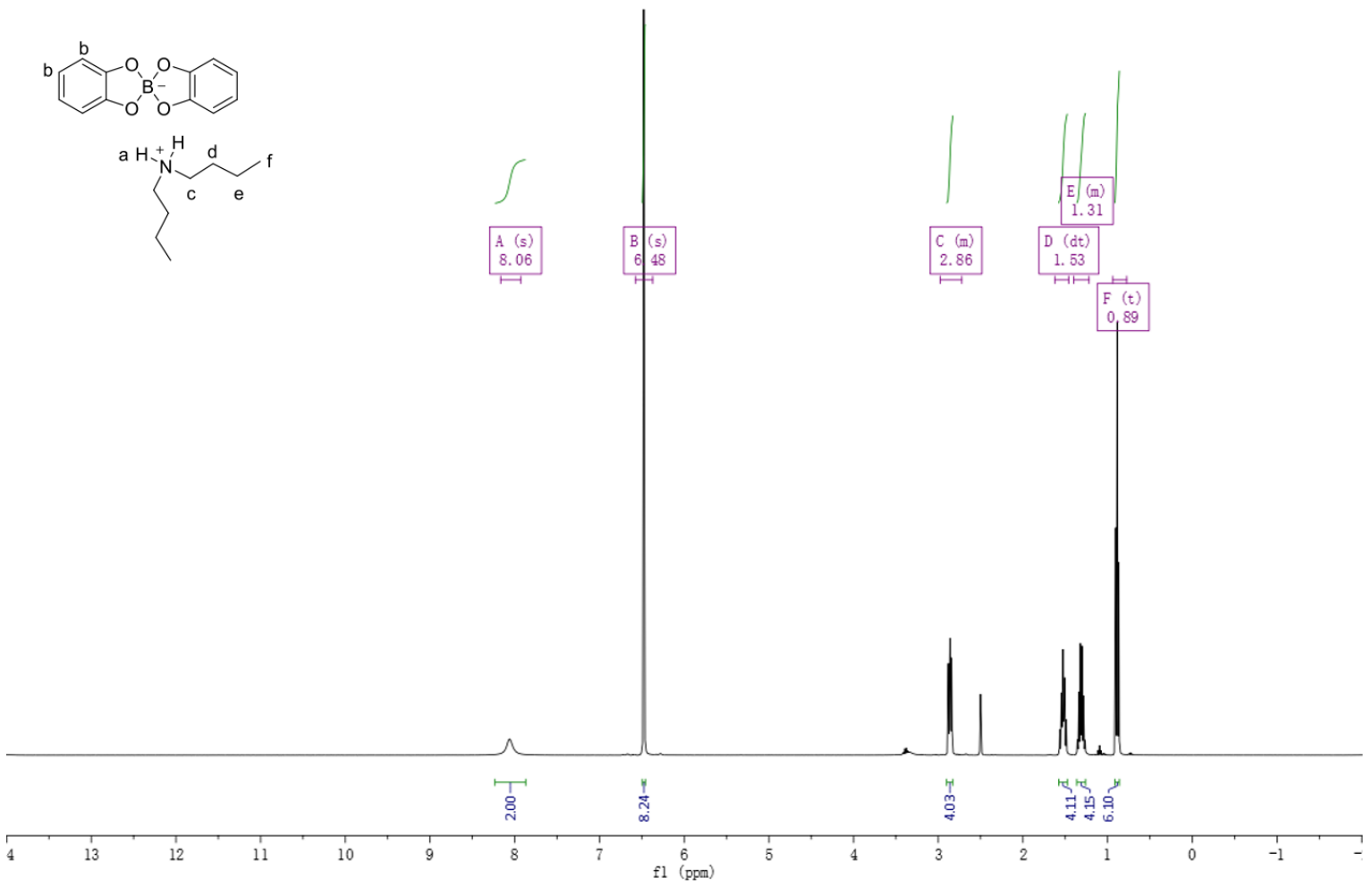

Figure S1. ${ }^{1} \mathrm{H}$ NMR spectra of model compound-DBA in DMSO- $d 6$.

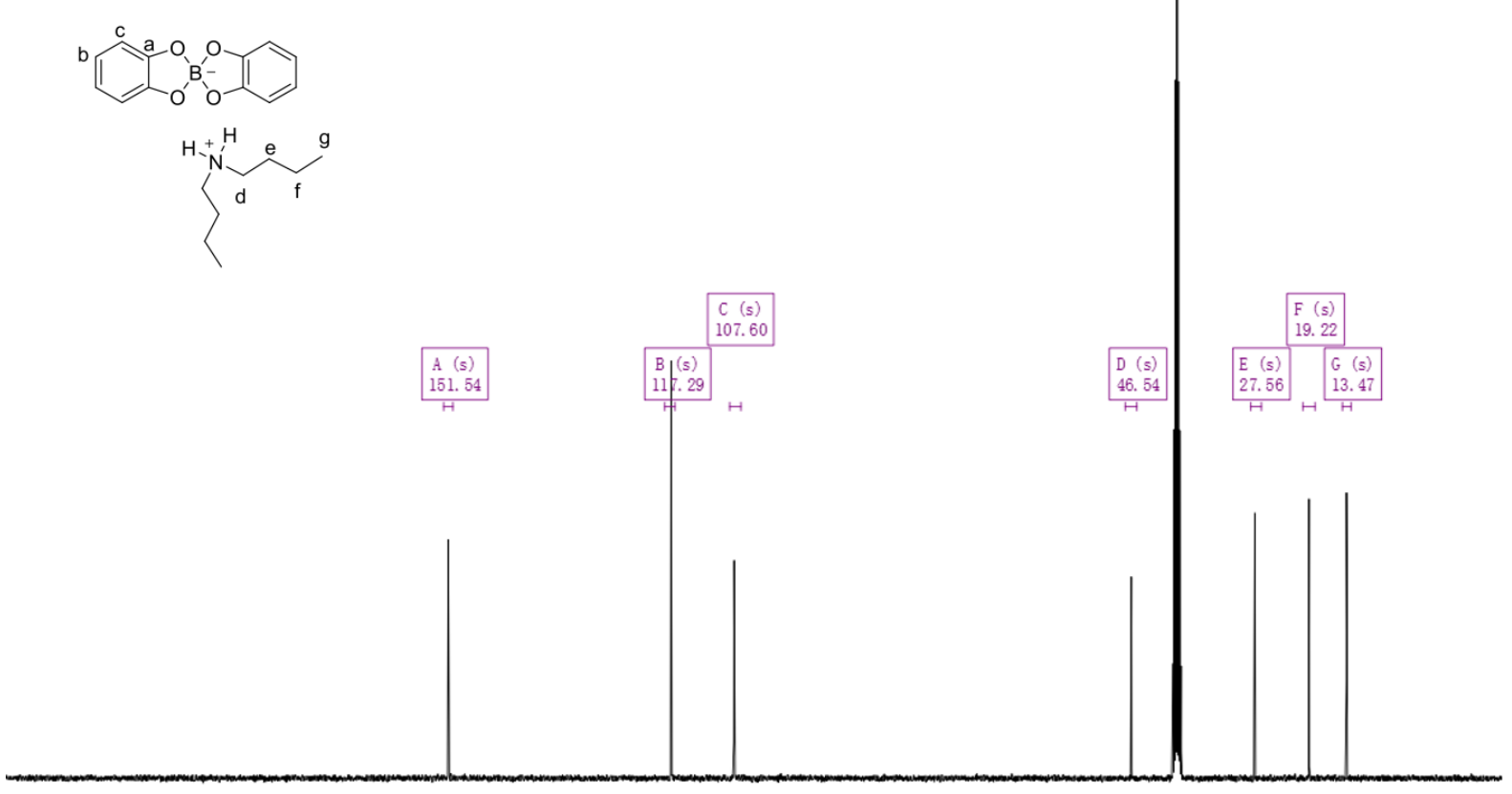

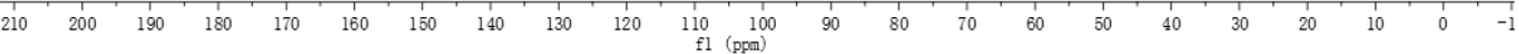

Figure S2. ${ }^{13} \mathrm{C}$ NMR spectra of model compound-DBA in DMSO- $d 6$. 


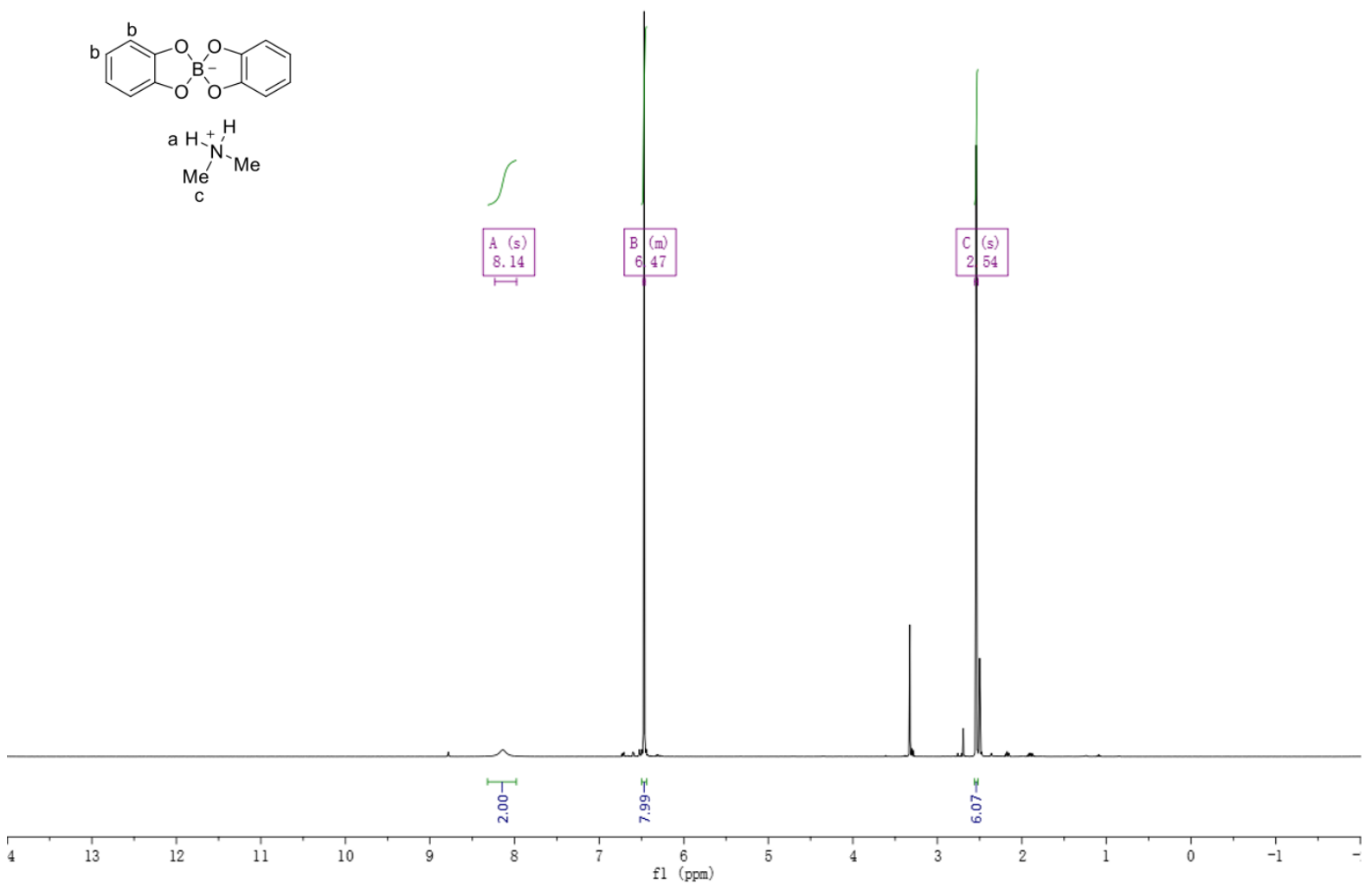

Figure S3. ${ }^{1} \mathrm{H}$ NMR spectra of model compound-DMA in DMSO- $d_{6}$.

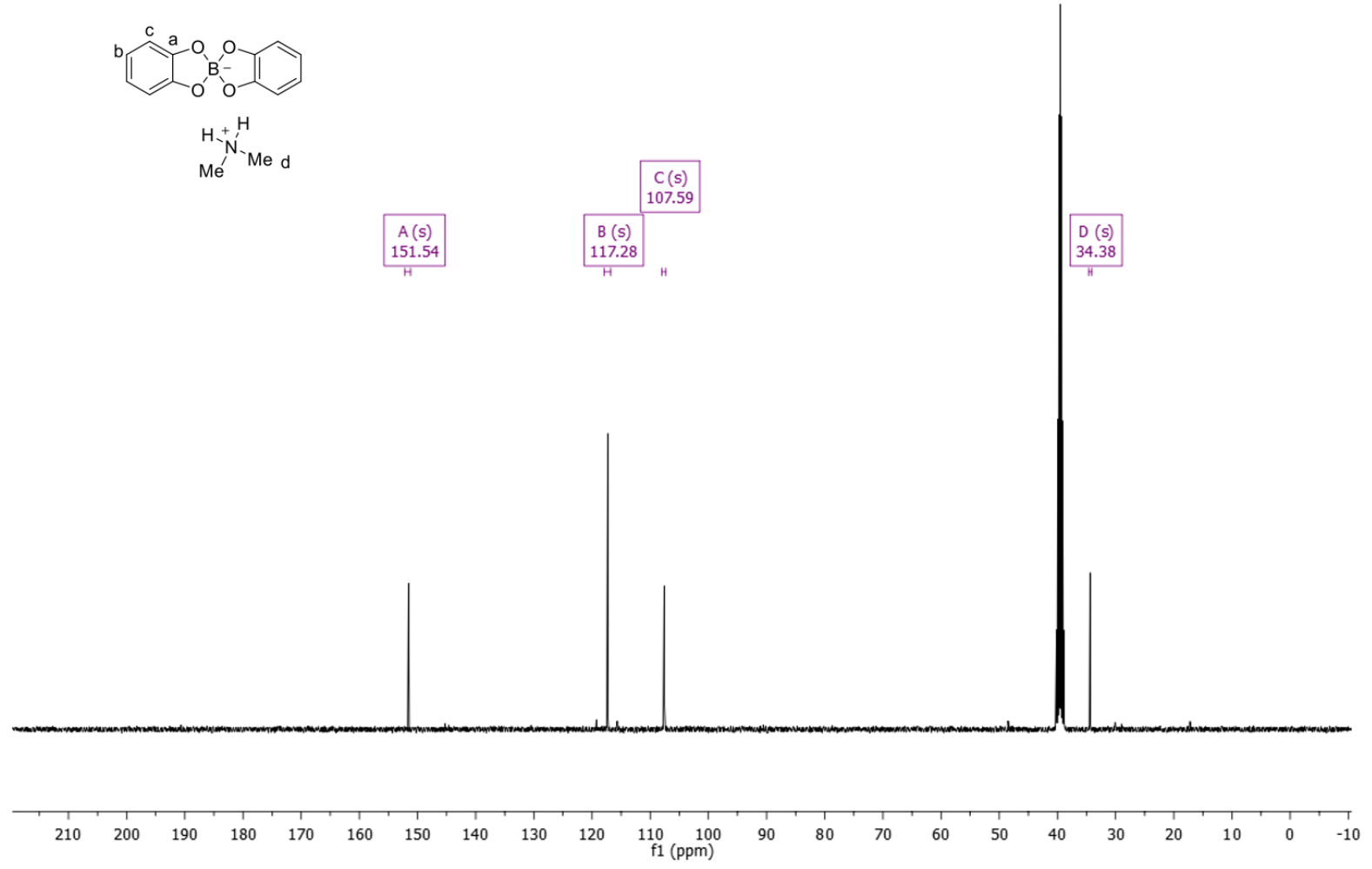

Figure S4. ${ }^{13} \mathrm{C}$ NMR spectra of model compound-DMA in DMSO- $d_{6}$. 


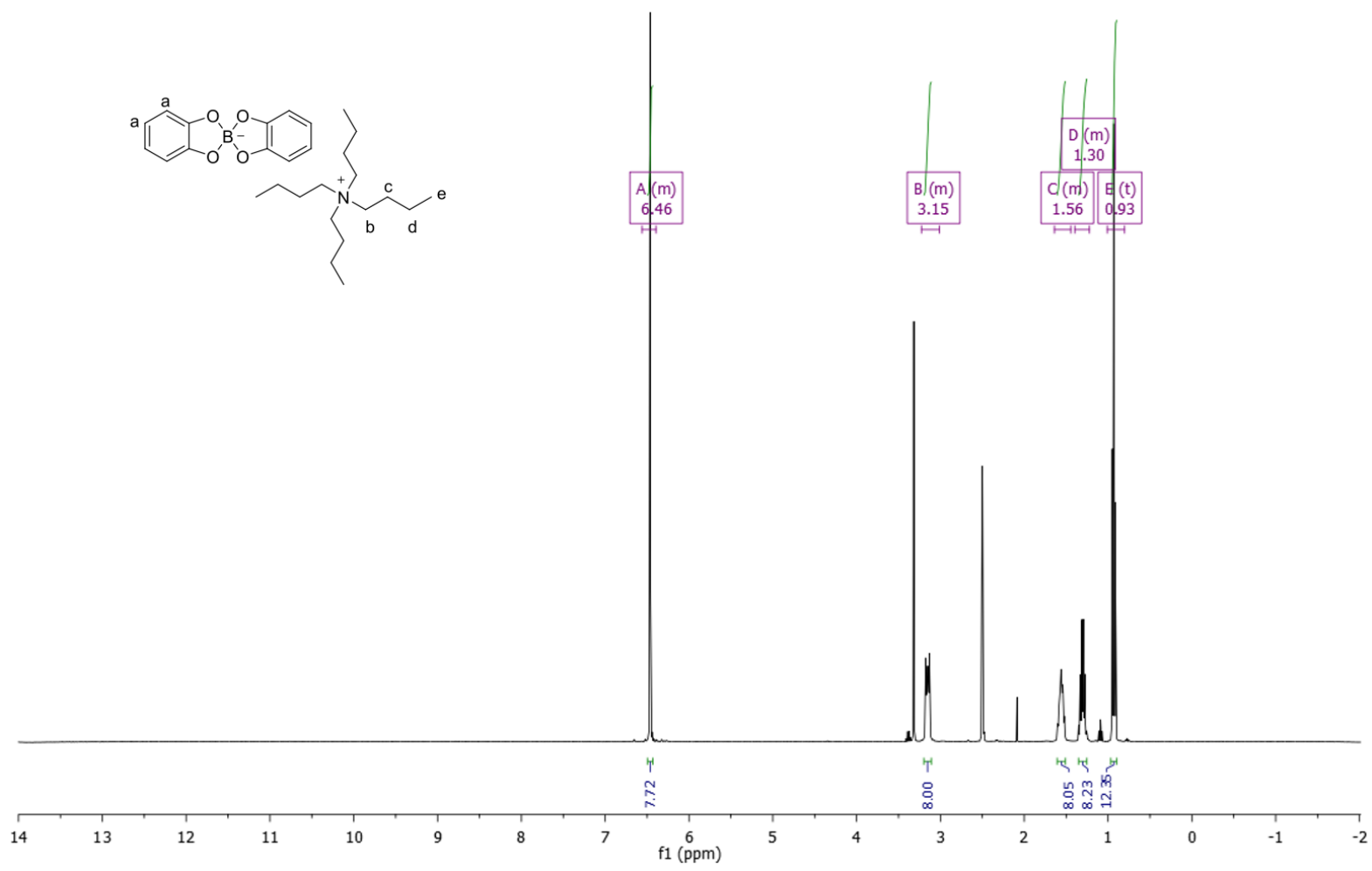

Figure S5. ${ }^{1} \mathrm{H}$ NMR spectra of model compound-TBA in DMSO- $d_{6}$.

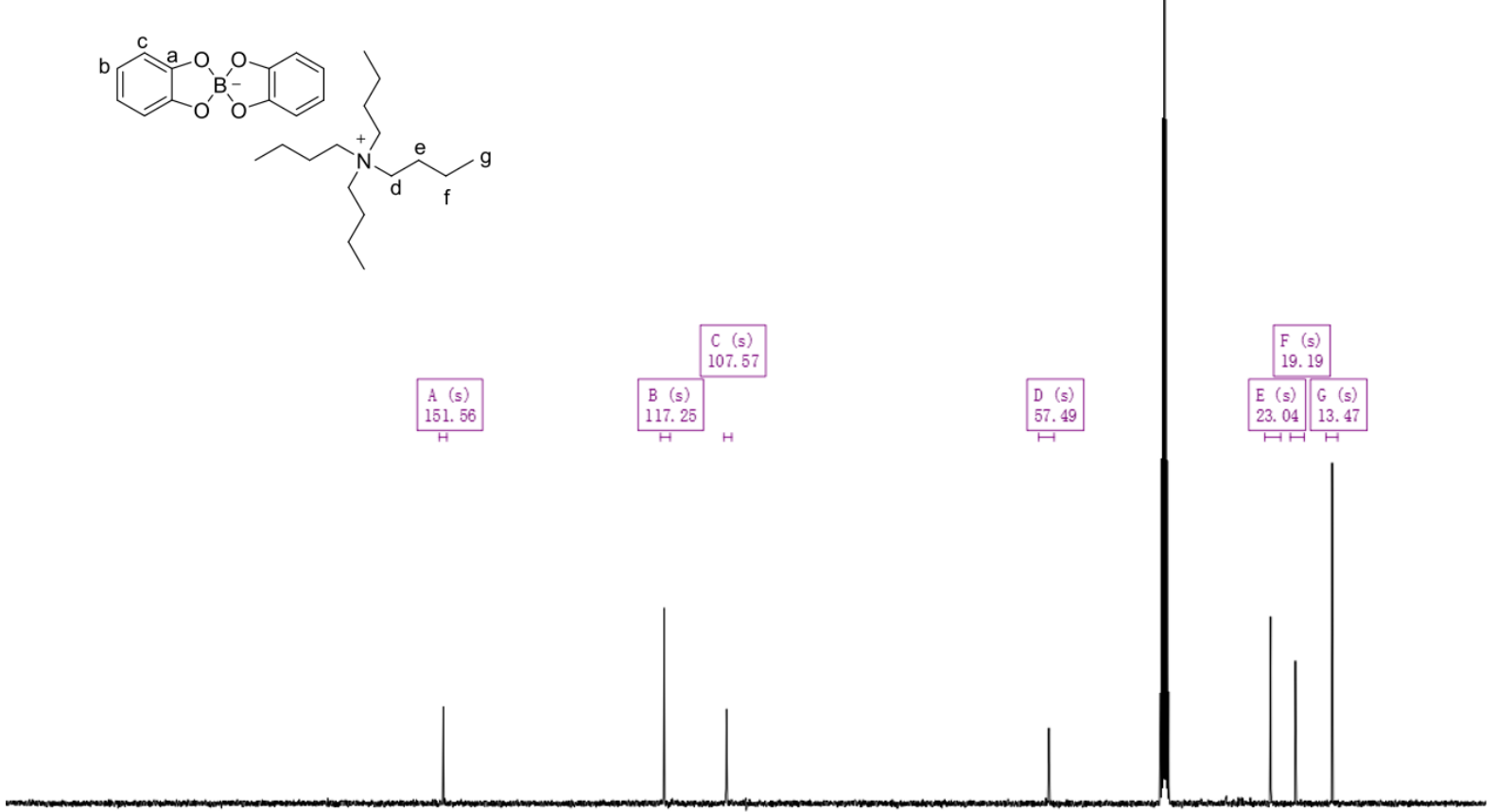

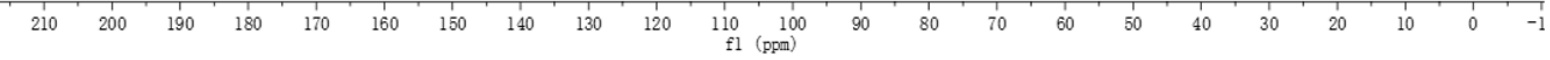

Figure S6. ${ }^{13} \mathrm{C}$ NMR spectra of model compound-TBA in DMSO- $d_{6}$. 


\section{Conformations reported for spiroborate units in the Cambridge Structural Database (CSD)}

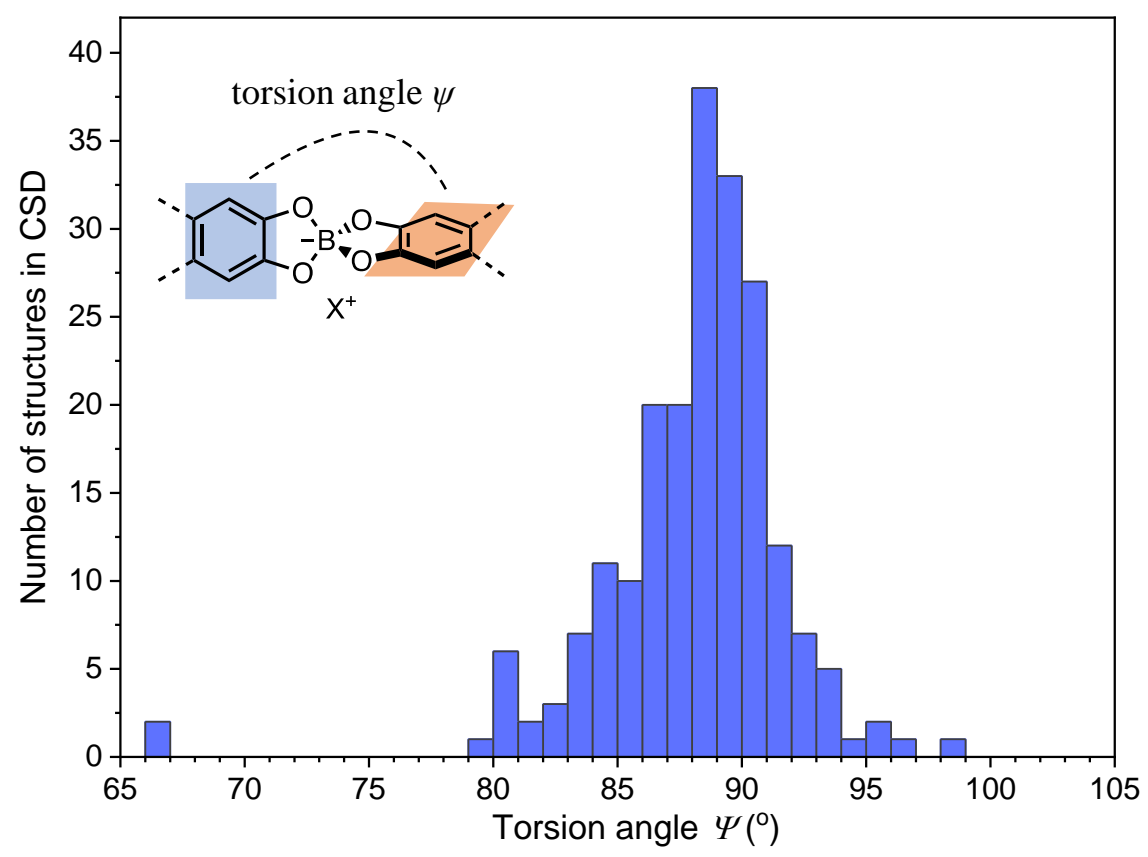

Figure S7. Distribution of the torsion angles expressed by spiroborate units as in the CSD at the time of submission; the most commonly reported angle is close to 90 degrees, supporting our reticular design strategy.

\section{Single Crystal Structure of Model Compounds}
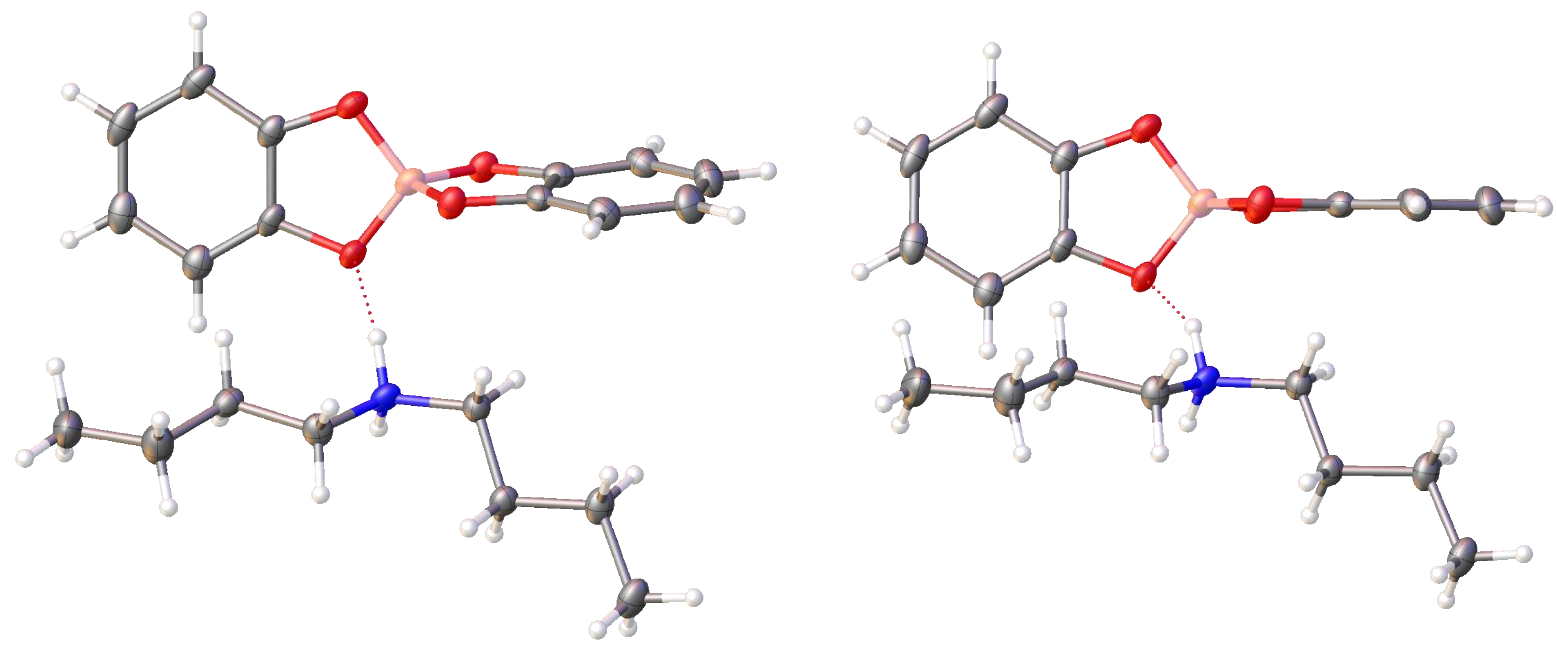

Figure S8. Displacement ellipsoid plots from the single crystal structure of model compound-DBA; two views are shown; ellipsoids are displayed at $50 \%$ probability level. $\mathrm{C}=$ grey; $\mathrm{H}=$ white; $\mathrm{N}=$ blue; $\mathrm{O}=$ red; $\mathrm{B}=$ pink. 
Table S2. Single crystal refinement details for model compound-DBA.

\begin{tabular}{|c|c|}
\hline Name & model compound-DBA \\
\hline Crystallization solvent & ethyl acetate \\
\hline Wavelength (Å) & $0.71073(\mathrm{Mo}-\mathrm{K} \alpha)$ \\
\hline Formula & $\mathrm{C}_{12} \mathrm{H}_{8} \mathrm{BO}_{4}, \mathrm{C}_{8} \mathrm{H}_{20} \mathrm{~N}$ \\
\hline $\operatorname{Mr}\left(\mathrm{g} \mathrm{mol}^{-1}\right)$ & 357.24 \\
\hline Crystal size (mm) & $0.41 \times 0.25 \times 0.13$ \\
\hline Crystal system & triclinic \\
\hline Space group & $P \overline{1}$ \\
\hline$a(\AA)$ & $8.1206(6)$ \\
\hline$b(\AA)$ & $10.1529(9)$ \\
\hline$c(\AA)$ & $12.4939(10)$ \\
\hline$V\left(\AA^{3}\right)$ & $102.267(7)$ \\
\hline$\rho_{\text {calcd }}\left(\mathrm{g} \mathrm{cm}^{-1}\right)$ & $104.960(7)$ \\
\hline $\mathbf{Z}$ & $93.689(6)$ \\
\hline $\mathbf{T}(\mathbf{K})$ & $964.68(14)$ \\
\hline$\mu\left(\mathrm{mm}^{-1}\right)$ & 1.230 \\
\hline$F(000)$ & 2 \\
\hline $2 \theta$ range $\left({ }^{\circ}\right)$ & $100.00(10)$ \\
\hline Reflections collected & 0.084 \\
\hline Independent reflections & 384.0 \\
\hline Data / restraints / parameters & $3.474-60.924$ \\
\hline $\boldsymbol{R}_{\text {int }}$ & 12044 \\
\hline Final $R_{1}$ values $(I>2 \sigma(I))$ & 4721 \\
\hline Final $R_{1}$ values (all data) & $4721 / 0 / 245$ \\
\hline$w R_{2}$ (all data) & 0.0376 \\
\hline Goodness-of-fit on $F^{2}$ & 0.0495 \\
\hline Crystallization solvent & 0.0772 \\
\hline Wavelength (A) & 0.1297 \\
\hline Formula & 1.052 \\
\hline
\end{tabular}



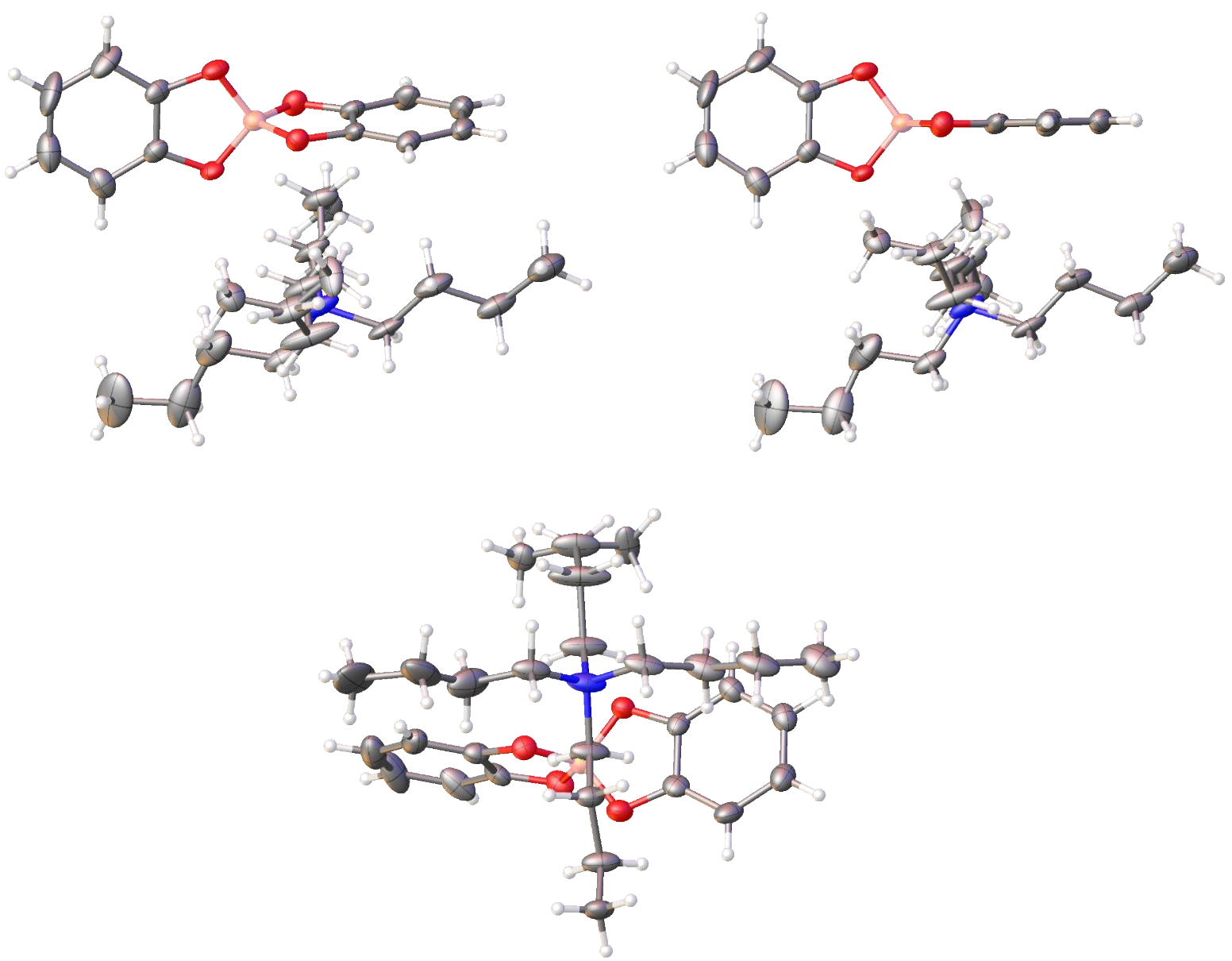

Figure S9. Displacement ellipsoid plots from the single crystal structure of model compound-TBA; three views are shown; ellipsoids are displayed at $50 \%$ probability level. $\mathrm{C}=$ grey; $\mathrm{H}=$ white; $\mathrm{N}=$ blue; $\mathrm{O}=$ red; $\mathrm{B}=$ pink. 
Table S3. Single crystal refinement details for model compound-TBA.

\begin{tabular}{|c|c|}
\hline Name & model compound-TBA \\
\hline Crystallization solvent & ethanol $+\mathrm{Et}_{2} \mathrm{O}$ or ethyl acetate $+\mathrm{Et}_{2} \mathrm{O}$ \\
\hline Wavelength (A) & $0.71073(\mathrm{Mo-K} \alpha)$ \\
\hline Formula & $\mathrm{C}_{12} \mathrm{H}_{8} \mathrm{BO}_{4}, \mathrm{C}_{16} \mathrm{H}_{35} \mathrm{~N}$ \\
\hline $\operatorname{Mr}\left(\mathrm{g} \mathrm{mol}^{-1}\right)$ & 468.44 \\
\hline Crystal size (mm) & $0.22 \times 0.197 \times 0.041$ \\
\hline Crystal system & orthorhombic \\
\hline Space group & $\mathrm{Pca}_{1}$ \\
\hline$a(\AA)$ & $15.5802(3)$ \\
\hline$b(\AA)$ & $8.4052(2)$ \\
\hline$c(\AA)$ & $20.6412(4)$ \\
\hline$V\left(\AA^{3}\right)$ & $2703.05(10)$ \\
\hline$\rho_{\text {calcd }}\left(\mathrm{g} \mathrm{cm}^{-1}\right)$ & 1.151 \\
\hline $\mathbf{Z}$ & 4 \\
\hline $\mathbf{T}(\mathrm{K})$ & $100.00(13)$ \\
\hline$\mu\left(\mathbf{m m}^{-1}\right)$ & 0.075 \\
\hline$F(000)$ & 1020.00 \\
\hline $2 \theta$ range $\left({ }^{\circ}\right)$ & $3.946-58.296$ \\
\hline Reflections collected & 61889 \\
\hline Independent reflections & 6998 \\
\hline Data / restraints / parameters & $6998 / 2 / 322$ \\
\hline$R_{\text {int }}$ & 0.0397 \\
\hline Final $R_{1}$ values $(I>2 \sigma(I))$ & 0.0881 \\
\hline Final $R_{1}$ values (all data) & 0.0970 \\
\hline$w R_{2}$ (all data) & 0.1742 \\
\hline Goodness-of-fit on $F^{2}$ & 1.145 \\
\hline
\end{tabular}




\section{Fourier-transform infrared spectroscopy}

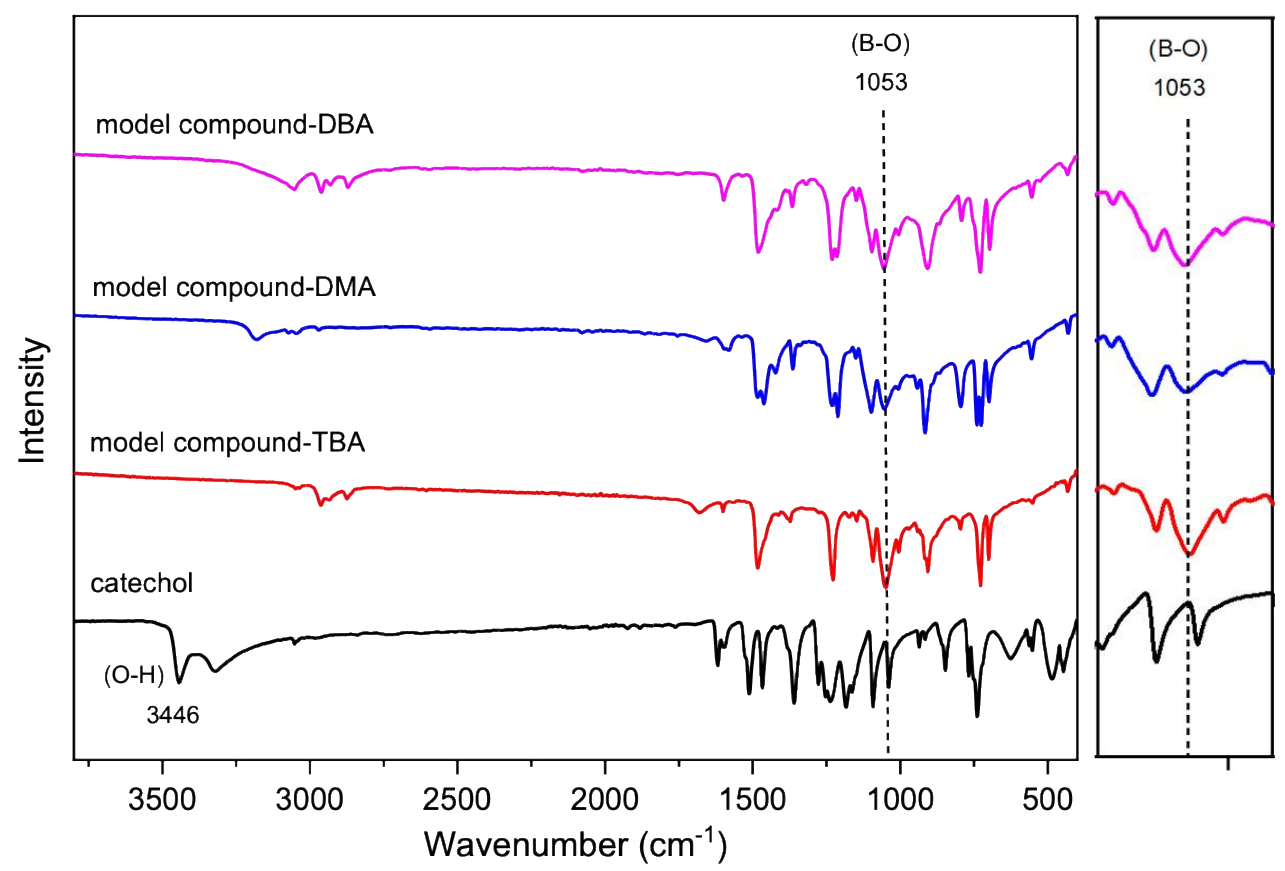

Figure S10. FT-IR spectra of catechol and model compounds.

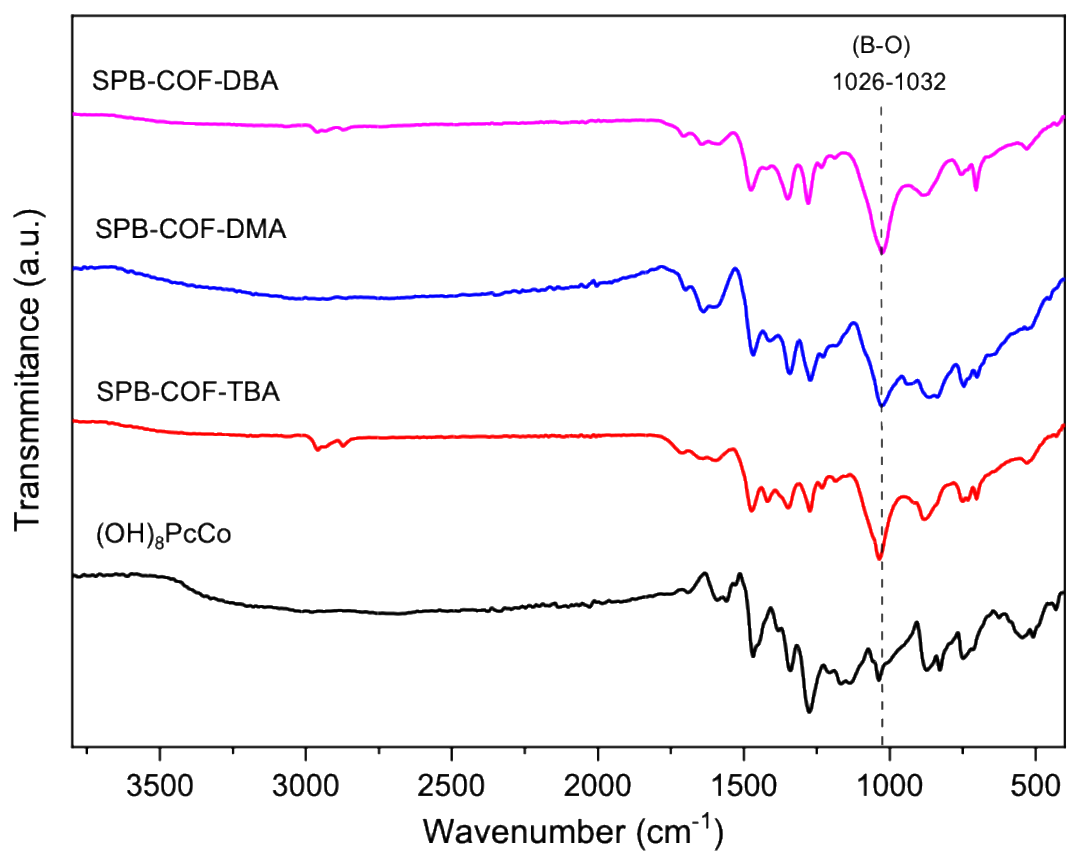

Figure S11. FT-IR spectra of $(\mathrm{OH})_{8} \mathrm{PcC}$ and SPB-COFs. 


\section{Solid state NMR spectra}

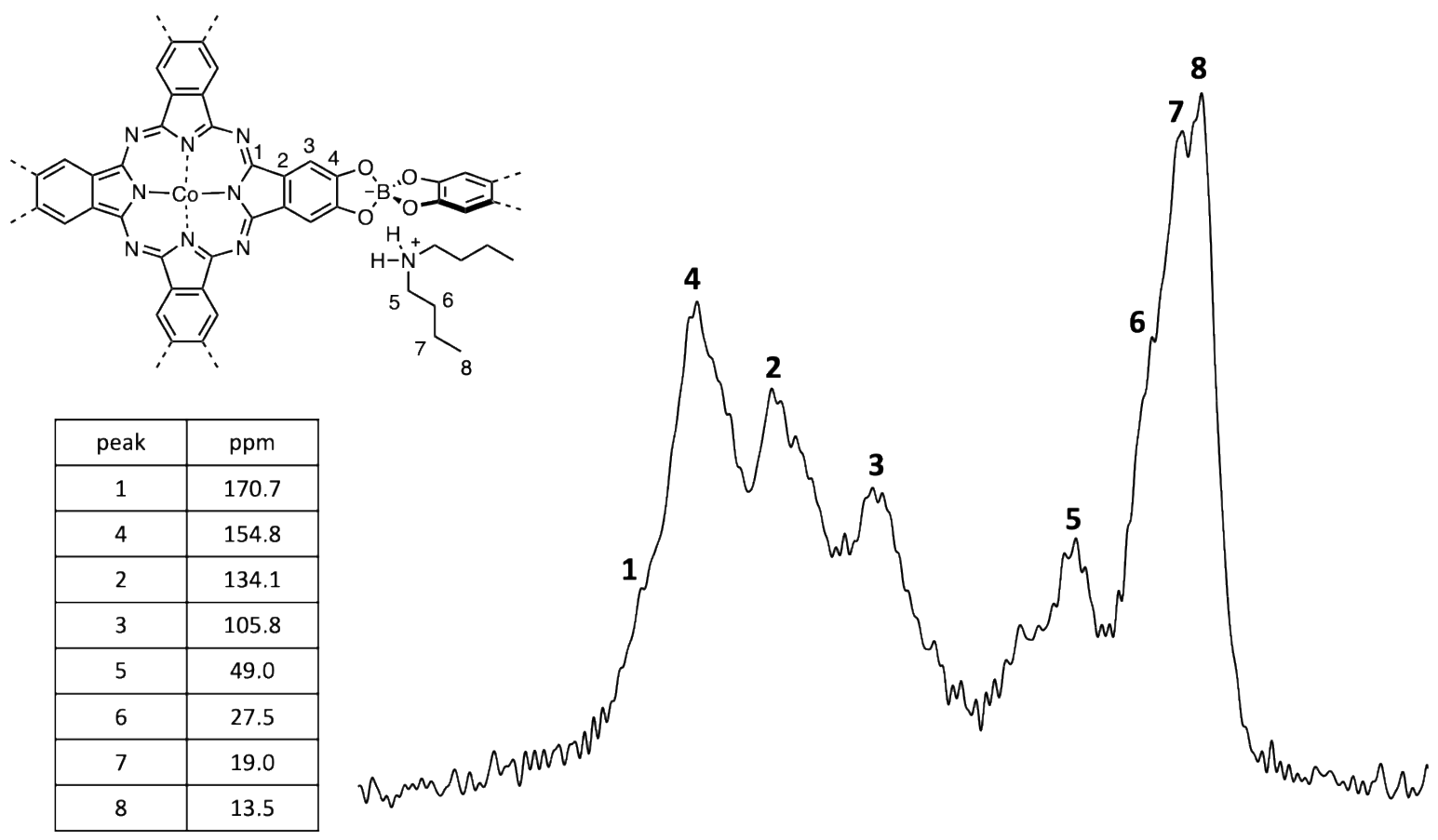

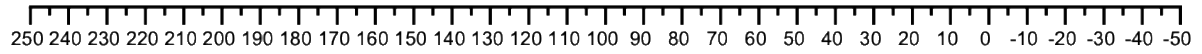
f1 (ppm)

Figure S12. Solid state ${ }^{13} \mathrm{C}$ CP/MAS NMR spectrum of SPB-COF-DBA. Supercritical $\mathrm{CO}_{2}$ activated COF was used for the measurement.
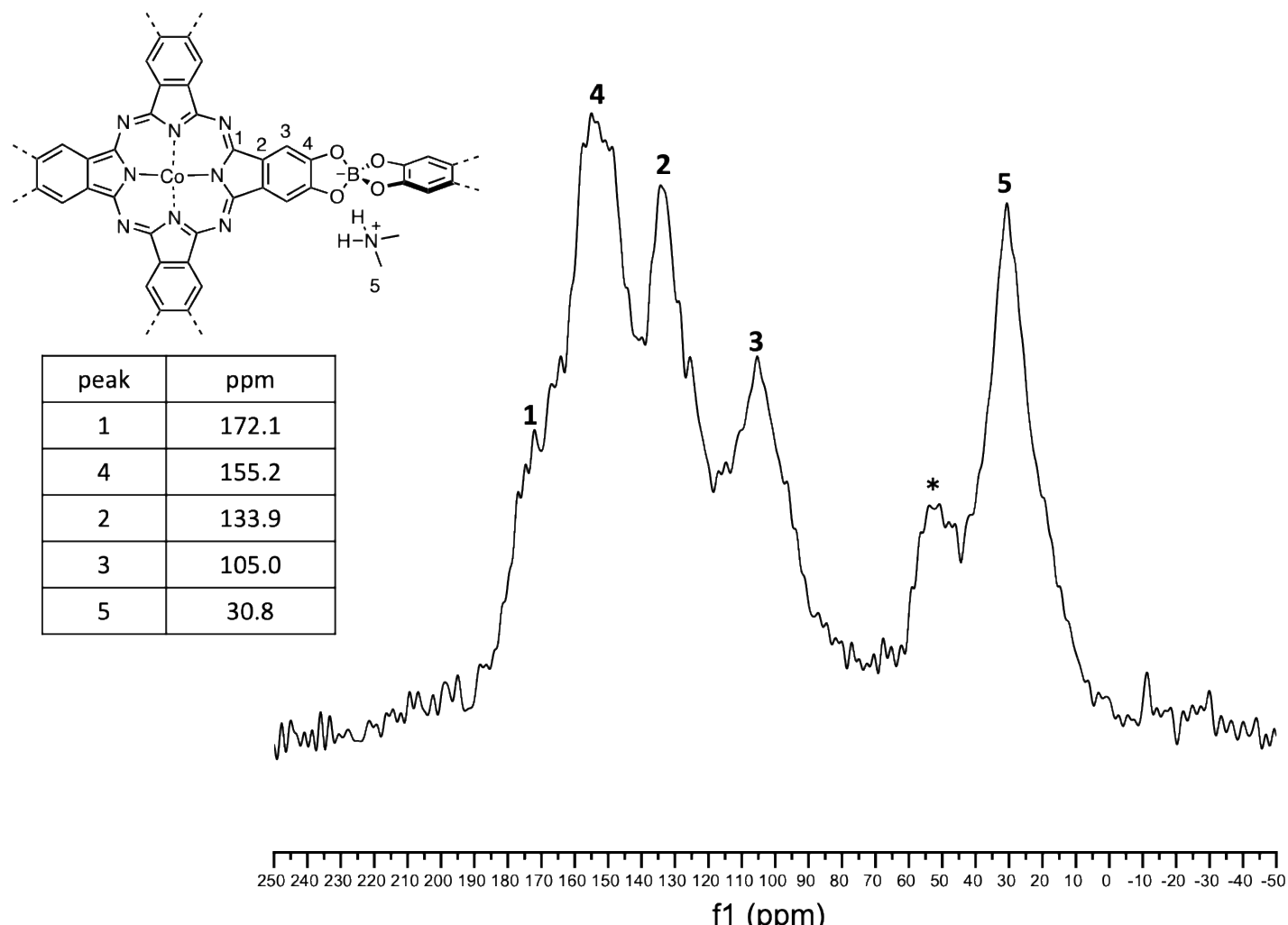

f1 (ppm)

Figure S13. Solid state ${ }^{13} \mathrm{C} \mathrm{CP} /$ MAS NMR spectrum of SPB-COF-DMA. Supercritical $\mathrm{CO}_{2}$ activated COF was used for the measurement. * Residual NMP. 


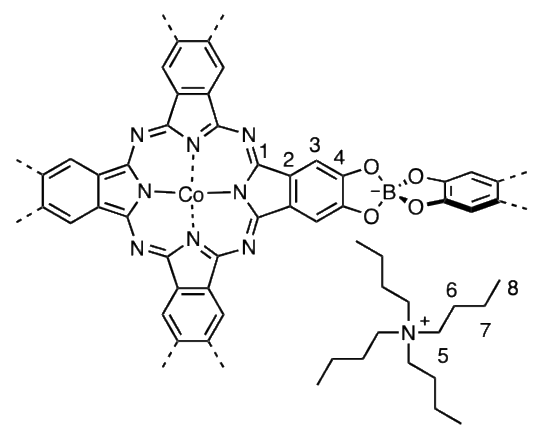

\begin{tabular}{|c|c|}
\hline peak & ppm \\
\hline 1 & 171.4 \\
\hline 4 & 151.6 \\
\hline 2 & 134.4 \\
\hline 3 & 102.4 \\
\hline 5 & 57.7 \\
\hline 6 & 21.2 \\
\hline 7 & 17.5 \\
\hline 8 & 14.1 \\
\hline
\end{tabular}
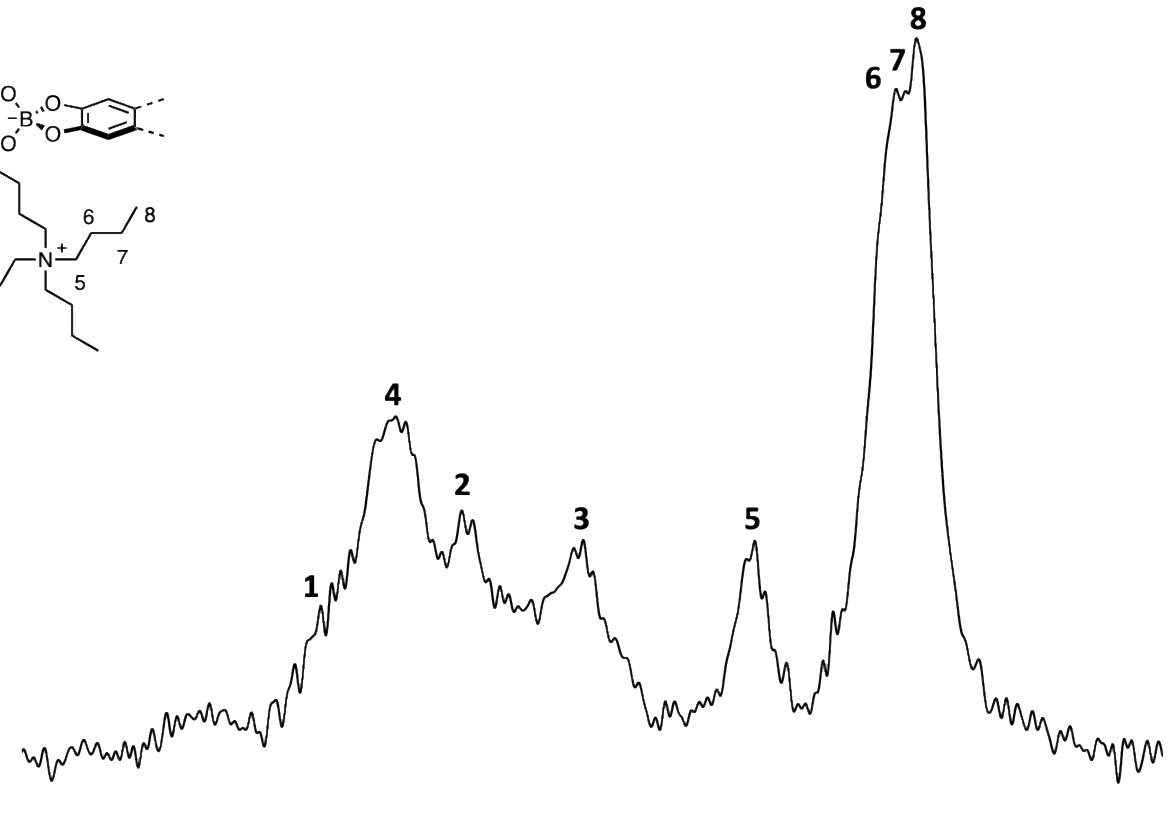

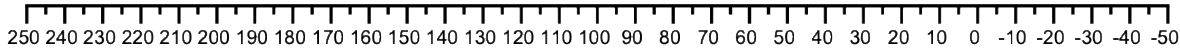
f1 (ppm)

Figure S14. Solid state ${ }^{13} \mathrm{C}$ CP/MAS NMR spectrum of SPB-COF-TBA. Supercritical $\mathrm{CO}_{2}$ activated COF was used for the measurement.

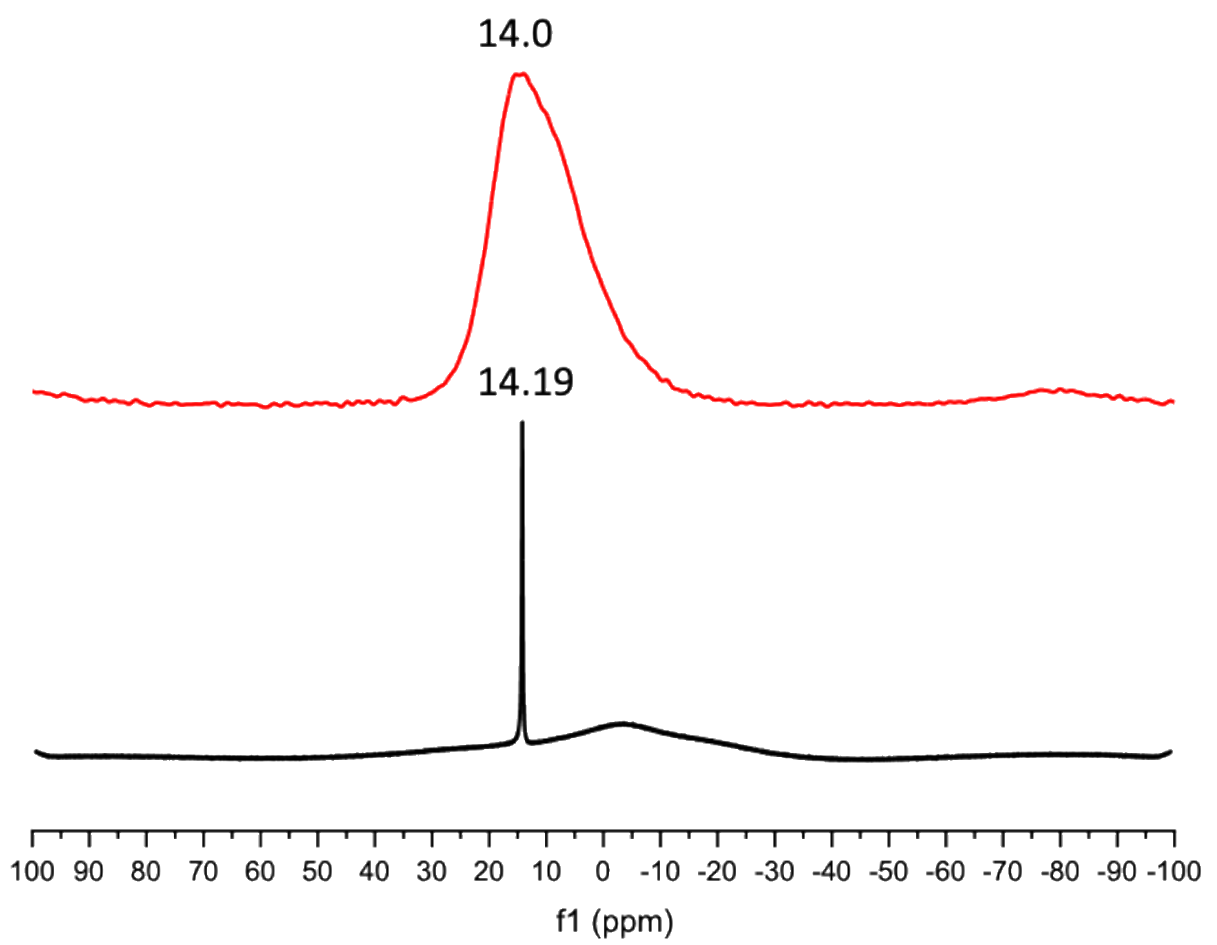

Figure S15. Solid-state ${ }^{11 B}$ MAS NMR spectrum of SPB-COF-DBA (red) compare with ${ }^{11}$ B solution NMR spectrum of model-compound-DBA (black). Supercritical $\mathrm{CO}_{2}$ activated $\mathrm{COF}$ was used for the measurement. 


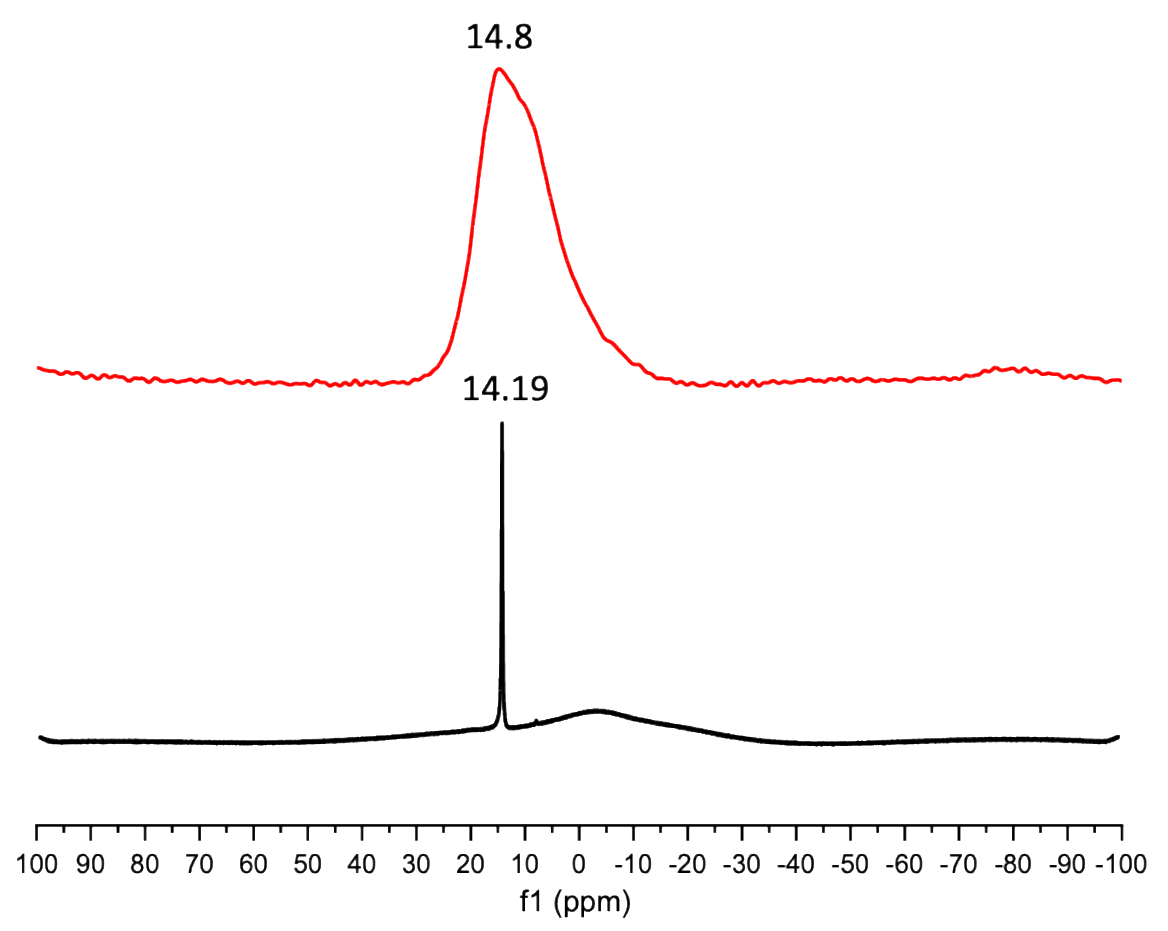

Figure S16. Solid-state ${ }^{11}$ B MAS NMR spectrum of SPB-COF-DMA (red) compare with ${ }^{11}$ B solution NMR spectrum of model-compound-DMA (black). Supercritical $\mathrm{CO}_{2}$ activated $\mathrm{COF}$ was used for the measurement.

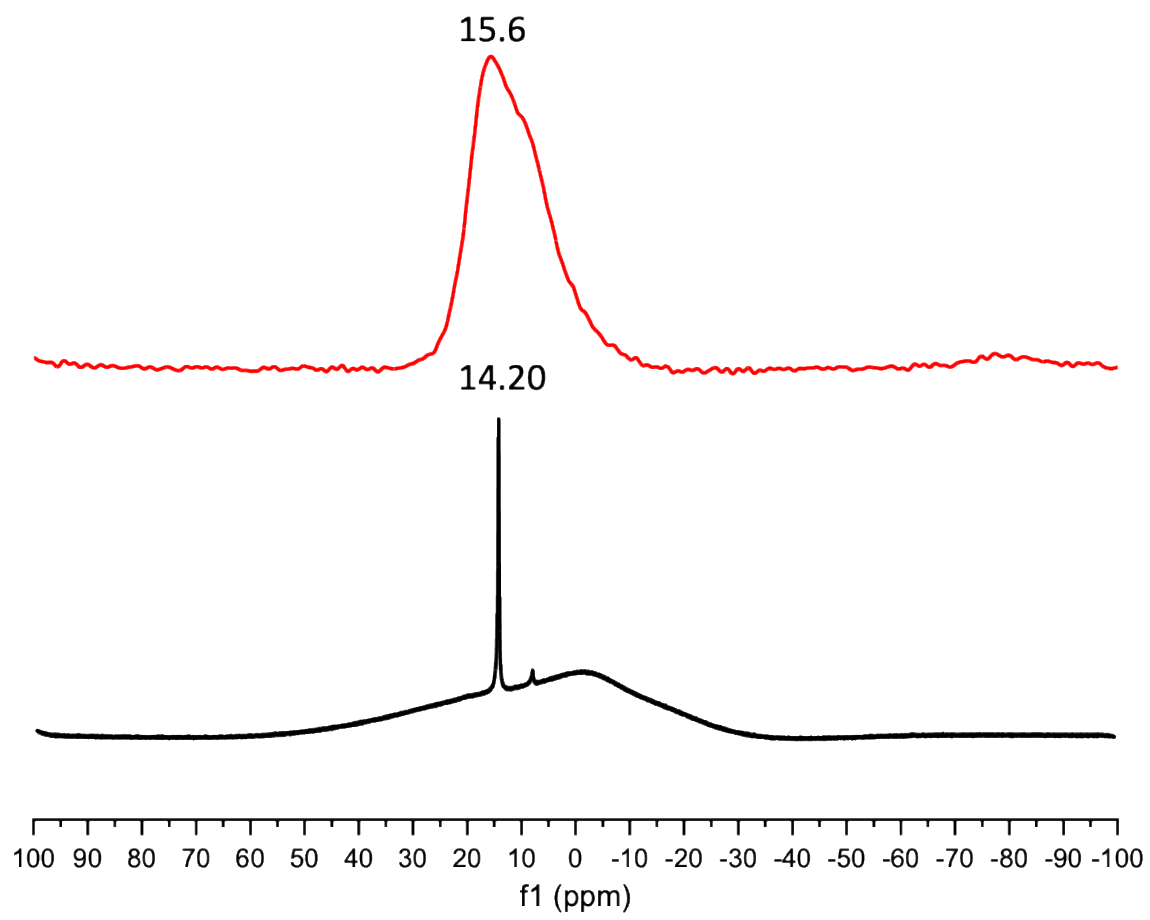

Figure S17. Solid-state ${ }^{11} \mathrm{~B}$ MAS NMR spectrum of SPB-COF-TBA (red) compare with ${ }^{11} \mathrm{~B}$ solution NMR spectrum of model-compound-TBA (black). Supercritical $\mathrm{CO}_{2}$ activated $\mathrm{COF}$ was used for the measurement. 
8. Thermogravimetric analysis

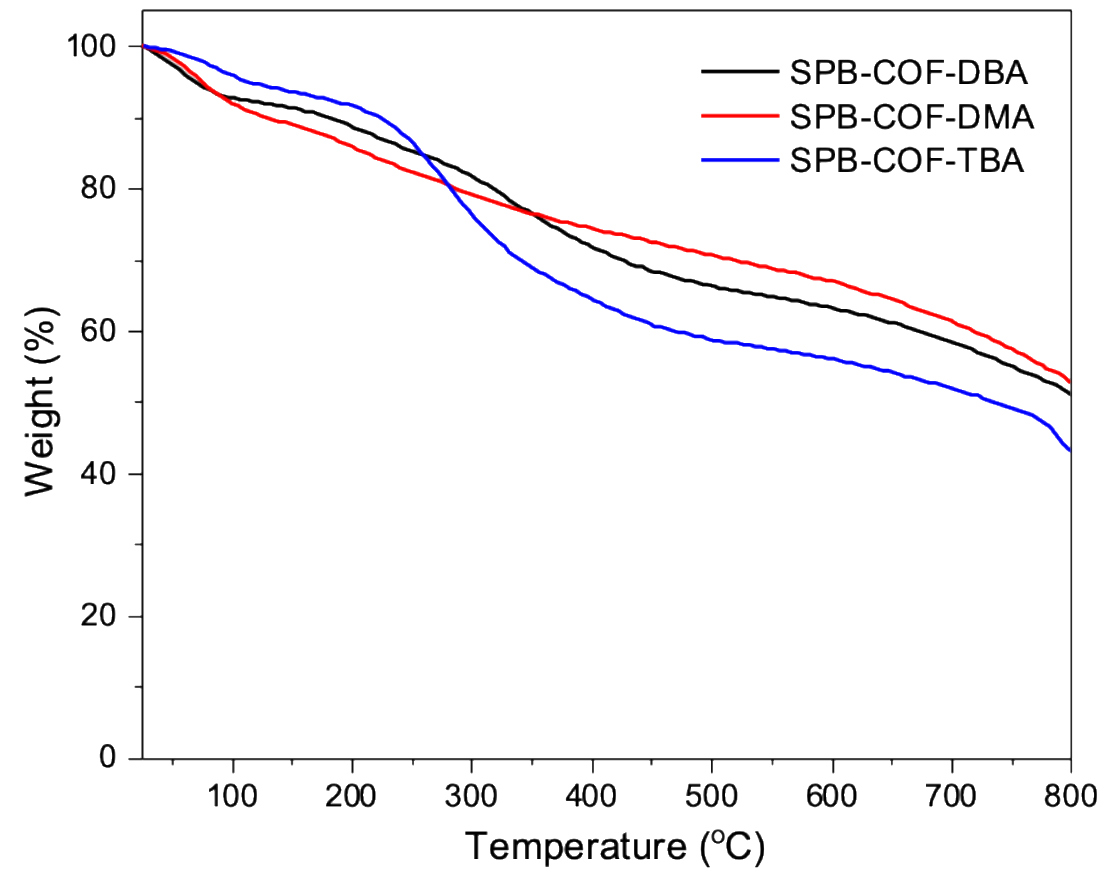

Figure S18. TGA curves of SPB-COFs in $\mathrm{N}_{2}$ atmosphere. 


\section{Scanning electron microscopy}
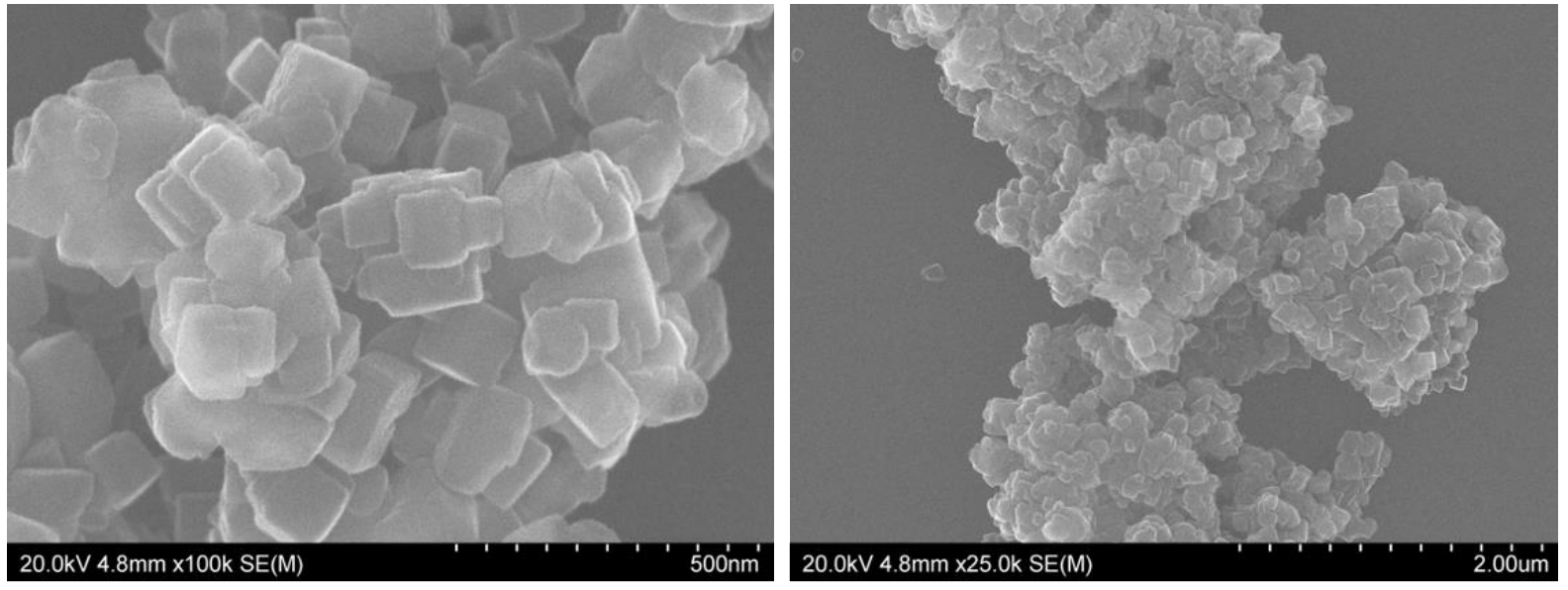

Figure S19. SEM images of SPB-COF-DBA. As-synthesized COF material was used for measurement.
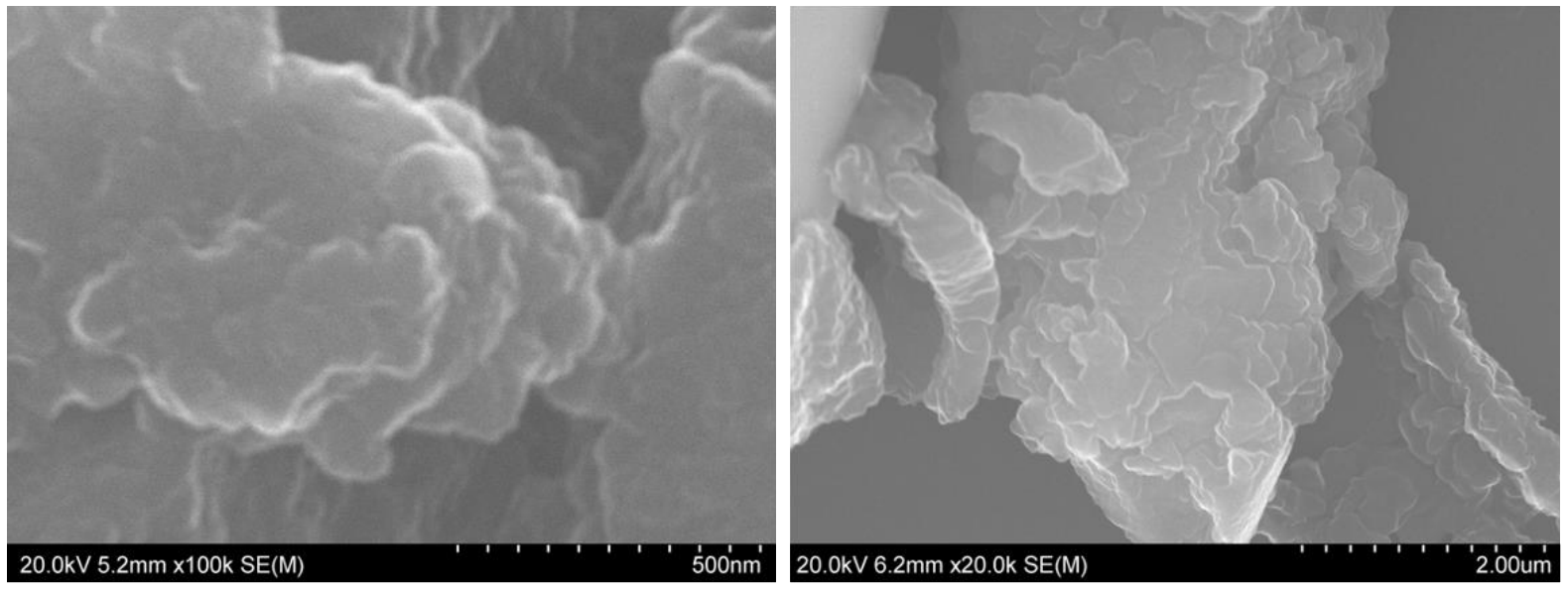

Figure S20. SEM images of SPB-COF-DMA. As-synthesized COF material was used for measurement.
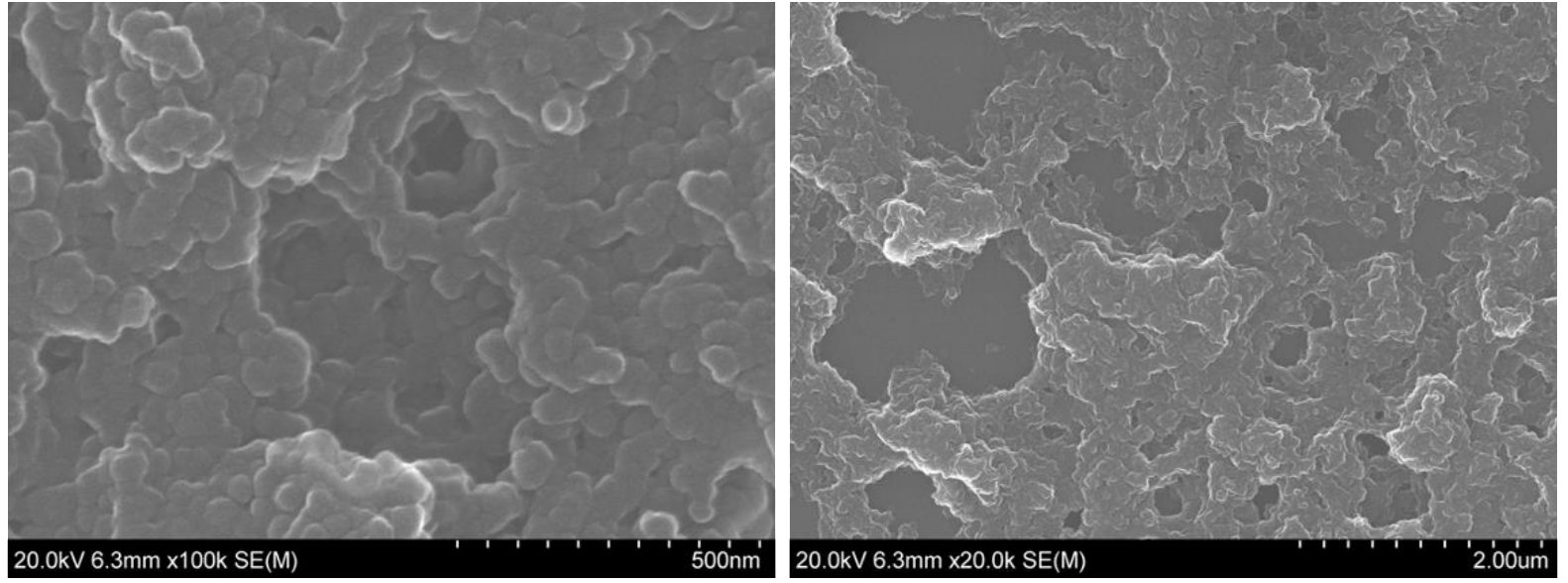

Figure S21. SEM images of SPB-COF-TBA. As-synthesized COF material was used for measurement. 


\section{Structure modelling}

According to the Reticular Chemistry Structure Resource (RCSR) database, ${ }^{6} 5$ topologies are possible for square-planar building blocks of a single type: edge-transitive nbo, lvt and rhr nets, and tcb and uks nets that have two edges. ${ }^{7}$ We constructed SPB-COF models for each of the five topologies and compared PXRD patterns simulated from the models with the experimental diffraction pattern. As shown in Figure S23, the experimental phase can be confirmed to be the nbo topology. Furthermore, we constructed SPB-COF models for two-fold and three-fold interpenetrated nbo nets. The comparison in Figure S25 reaffirms that the experimental phase is of the non-interpenetrated nbo topology.

For all the COF models included in Figures S23 and S25, we used spiro-orthocarbonate (SPC) linkages, instead of spiroborate (SPB) linkages, to connect the cobalt(II) phthalocyanine (PcCo) units. In doing so, we rendered all the frameworks charge neutral, hence allowing for their geometry optimizations without having to consider the counter ions. All the geometry optimizations made use of the universal force field, implemented in the Forcite module of the BIOVIA Materials Studio software. We also fully relaxed an SPBCOF model, with charge-balancing lithium cations, for the nbo topology, using density functional theory (DFT). As shown in Figure S26, the SPC-based model and the SPB-based model, both for the nbo topology, yielded almost identical PXRD patterns.

Periodic DFT calculations were carried out within the plane-wave pseudopotential formalism, using the Vienna ab initio Simulation Package code. ${ }^{8}$ A kinetic-energy cutoff of $500 \mathrm{eV}$ was used to define the planewave basis set, and the electronic Brillouin zone was integrated using $\Gamma$-centred Monkhorst-Pack grids with the smallest allowed spacing between k-points (KSPACING) being $0.25 \AA^{-1}$. Geometry optimizations, involving both lattice parameters and atomic positions, were performed employing the Perdew-Burke-Ernzerhof exchange-correlation functional with the DFT-D3(BJ) dispersion correction. Tolerances of $10^{-6} \mathrm{eV}$ and $10^{-2} \mathrm{eV} \AA^{-1}$ were applied during the optimization of the Kohn-Sham wavefunctions and the geometry optimizations, respectively. 
a)

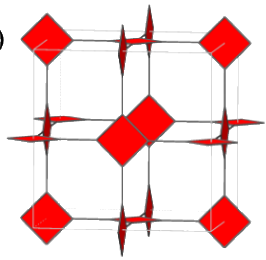

nbo

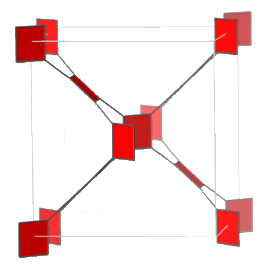

lvt

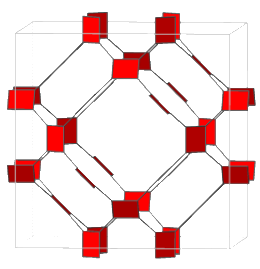

rhr

b)

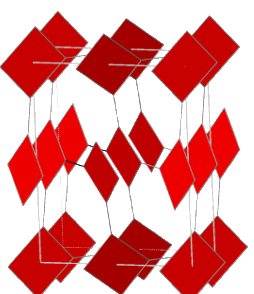

tcb

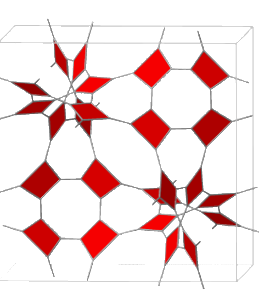

uks

Figure S22. Possible topologies of SPB-COFs: (a) edge-transitive nets; (b) nets with two edges. Grey lines indicate the unit cell.

Table S4. Structural and topological features of the topologies shown in Figure S22.

\begin{tabular}{|c|c|c|c|c|}
\hline Topology & Kinds of vertices & No. of edges & Pore diameter a $\AA$ & Space group of COF model \\
\hline nbo & 1 & 1 & 30.7 & Im-3m (No.229) \\
\hline lvt & 1 & 1 & 29.3 & I41/amd (No.141) \\
\hline rhr & 1 & 1 & 48.6 & Im-3m (No.229) \\
\hline uks & 1 & 2 & 54.5 & P1 (No.1) \\
\hline tcb & 1 & 2 & 13.1 & P1 (No.1) \\
\hline
\end{tabular}

a. Calculated using zeo++., ${ }^{9,10}$

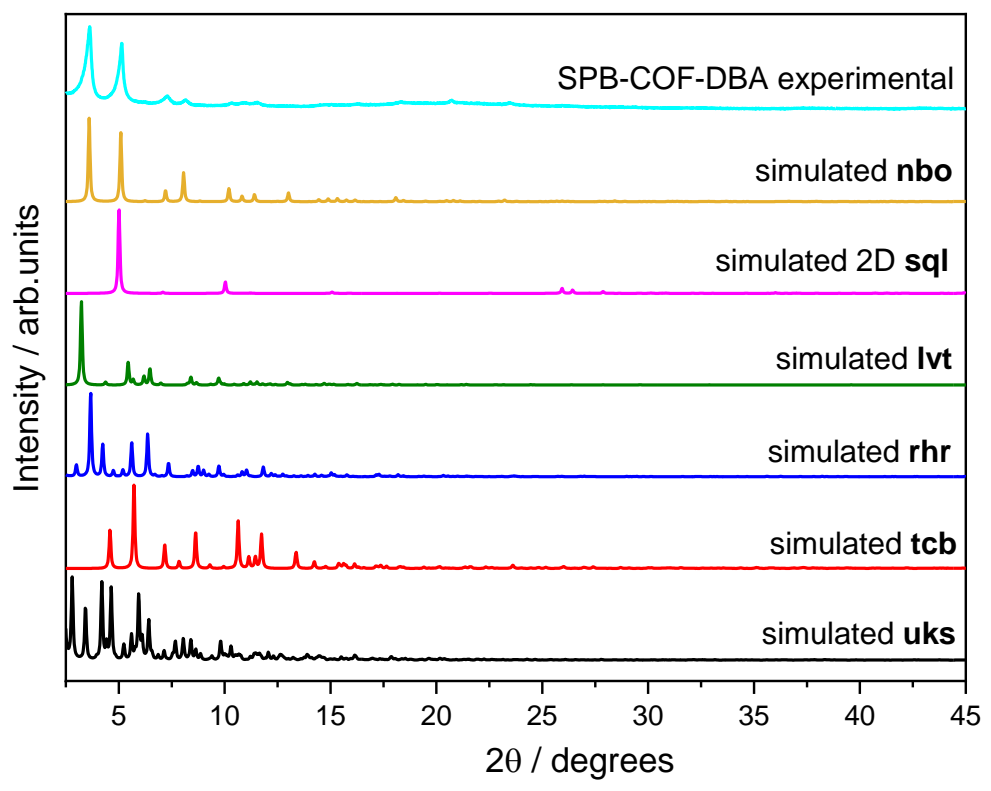

Figure S23. Comparison of the experimental PXRD pattern for SPB-COF-DBA and simulated PXRD patterns of the models based on the 3D nbo, lvt, rhr, tcb and uks topologies, and the 2D sql topology. 
a)

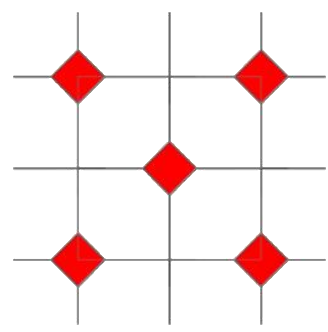

b)

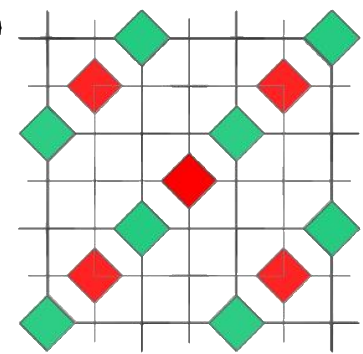

c)

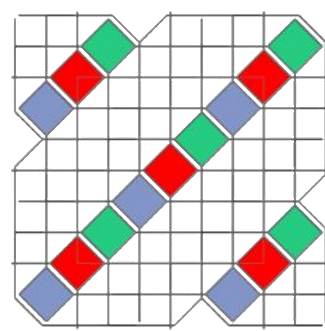

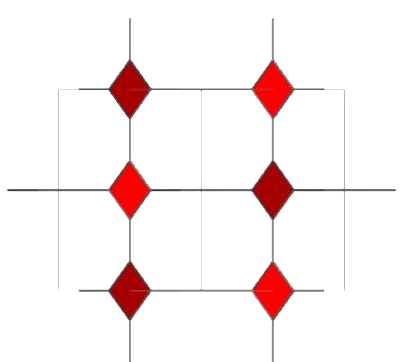
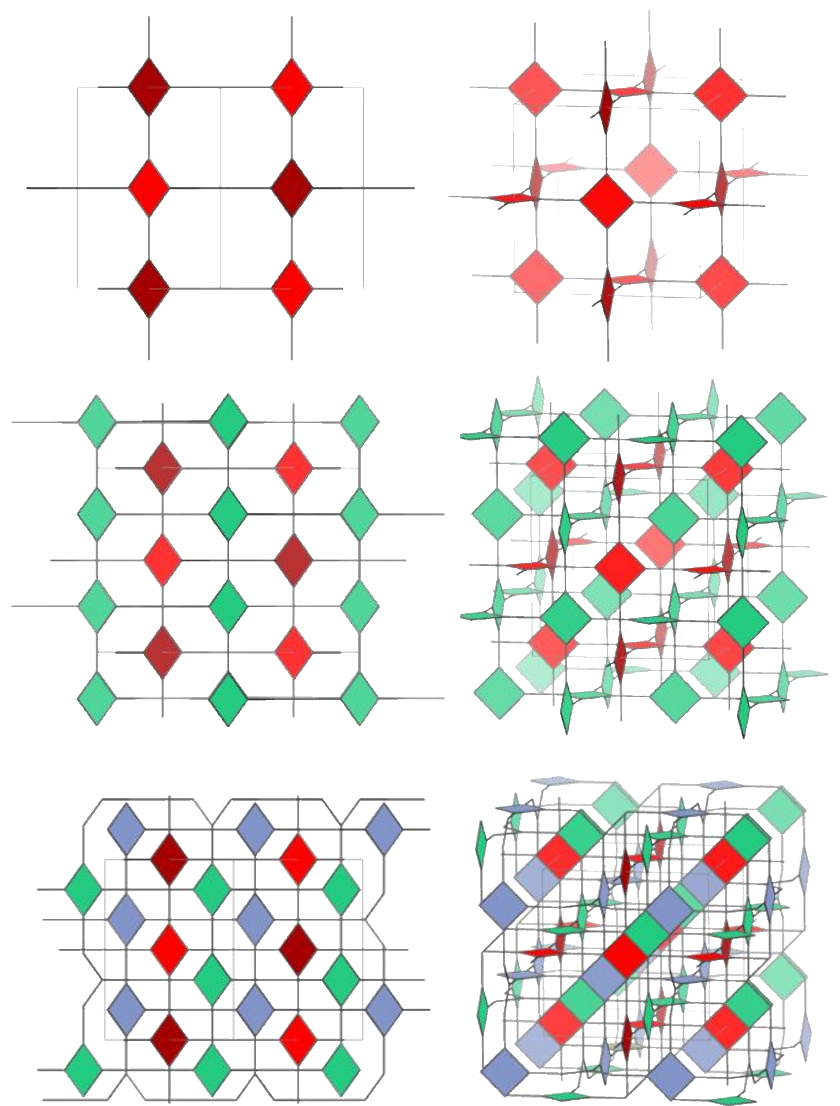

Figure S24. Three comparable views of (a) non-interpenetrated nbo net; (b) 2 -fold interpenetrated nbo net and (c) 3fold interpenetrated nbo net. PcCo nodes belonging to first, second or third fold are coloured in red, green or violet, respectively.

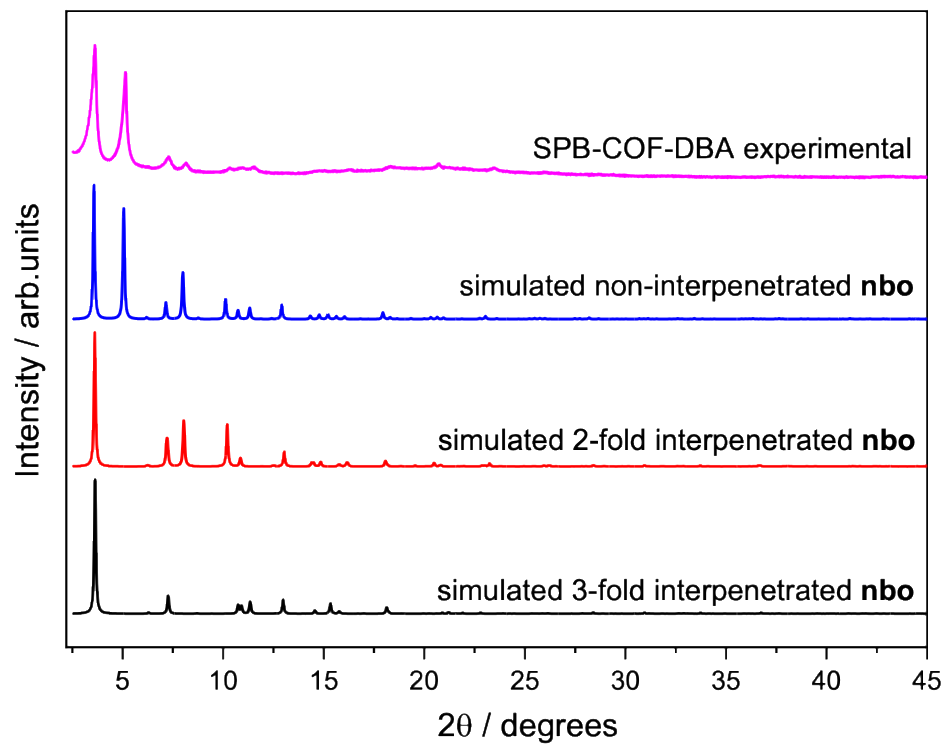

Figure S25. Comparison of the experimental PXRD pattern and simulated patterns for the nbo topology with the different degrees of interpenetration. 


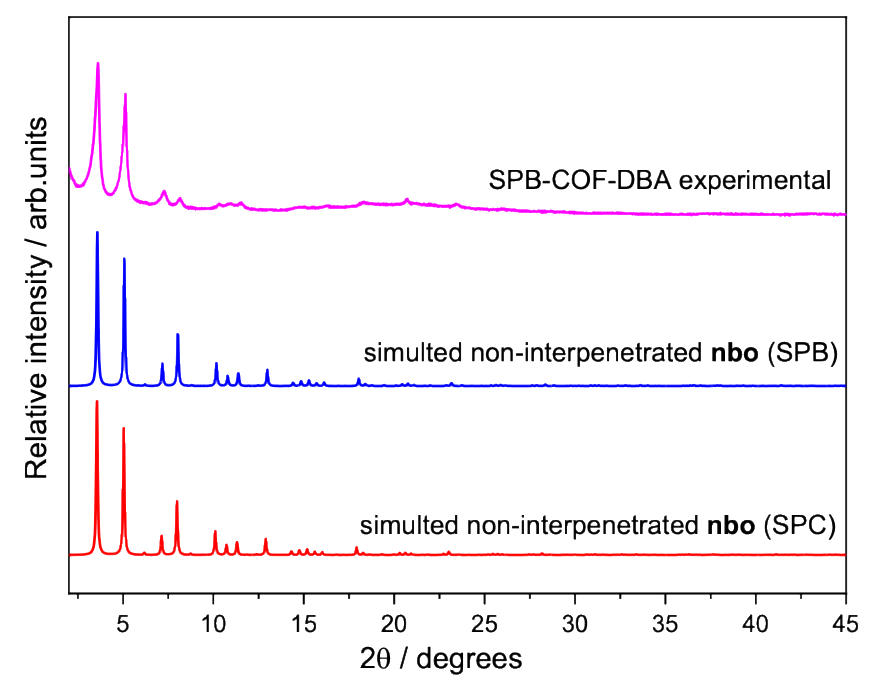

Figure S26. Comparison of the experimental PXRD pattern and simulated patterns of the models based on the spiroborate (SPB) or spiro-orthocarbonate (SPC) linkage.

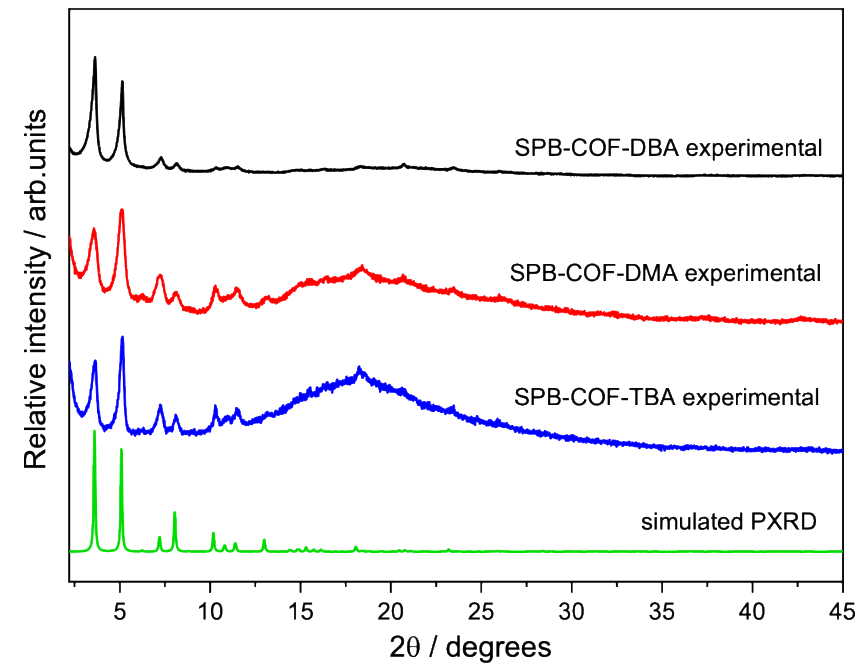

Figure S27. Experimental PXRD patterns for the as-synthesized SPB-COF-DBA, SPB-COF-DMA and SPB-COF-TBA. 


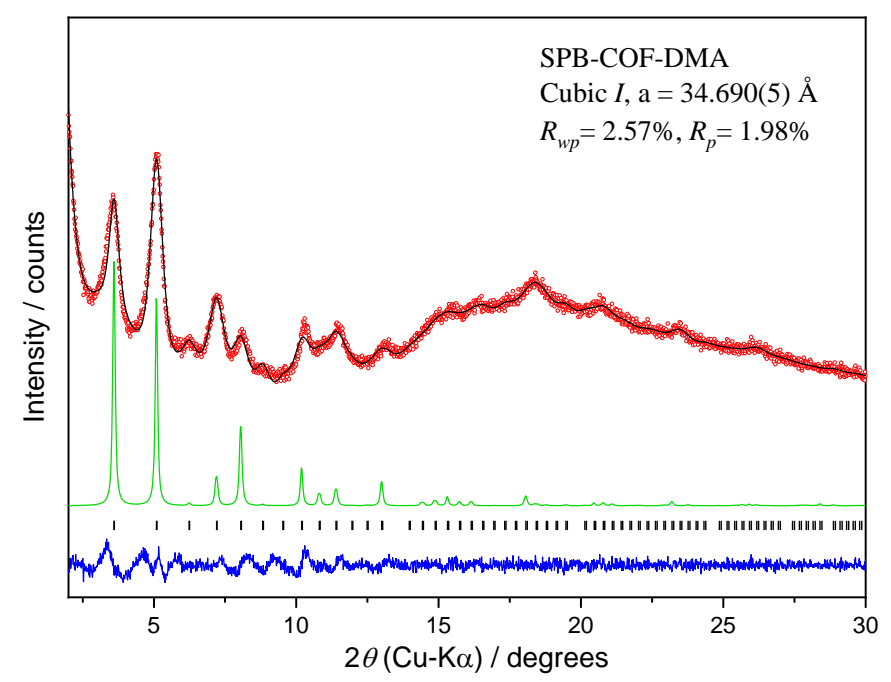

Figure S28. Experimental PXRD pattern (red), profile calculated from Pawley refinement (black) and residual (blue), and pattern simulated from the structural model (green) for SPB-COF-DMA. Reflection positions are shown by tick marks.

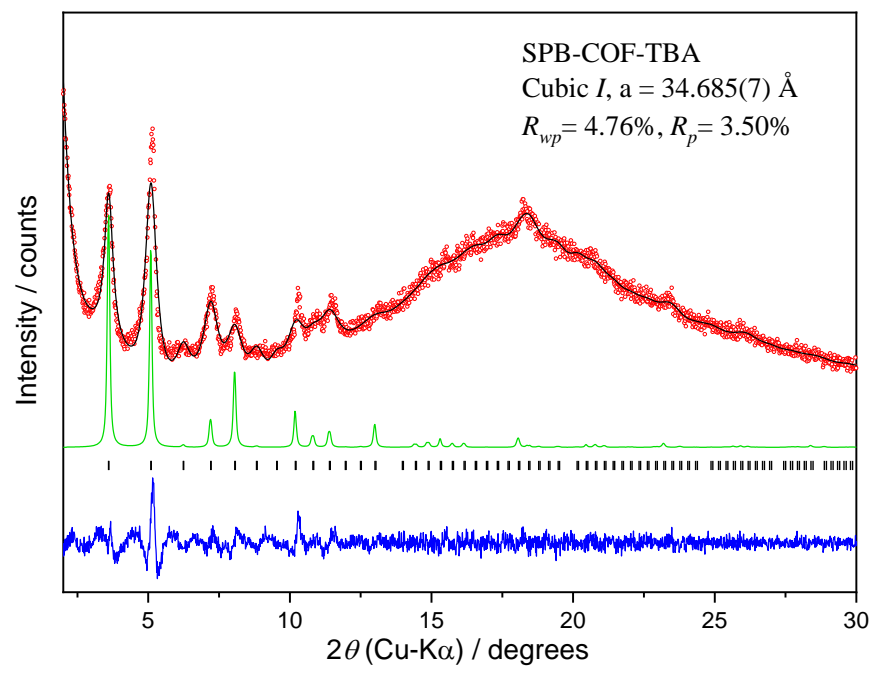

Figure S29. Experimental PXRD pattern (red), profile calculated from Pawley refinement (black) and residual (blue), and pattern simulated from the structural model (green) for SPB-COF-TBA. Reflection positions are shown by tick marks. 


\section{Transmission electron microscopy}

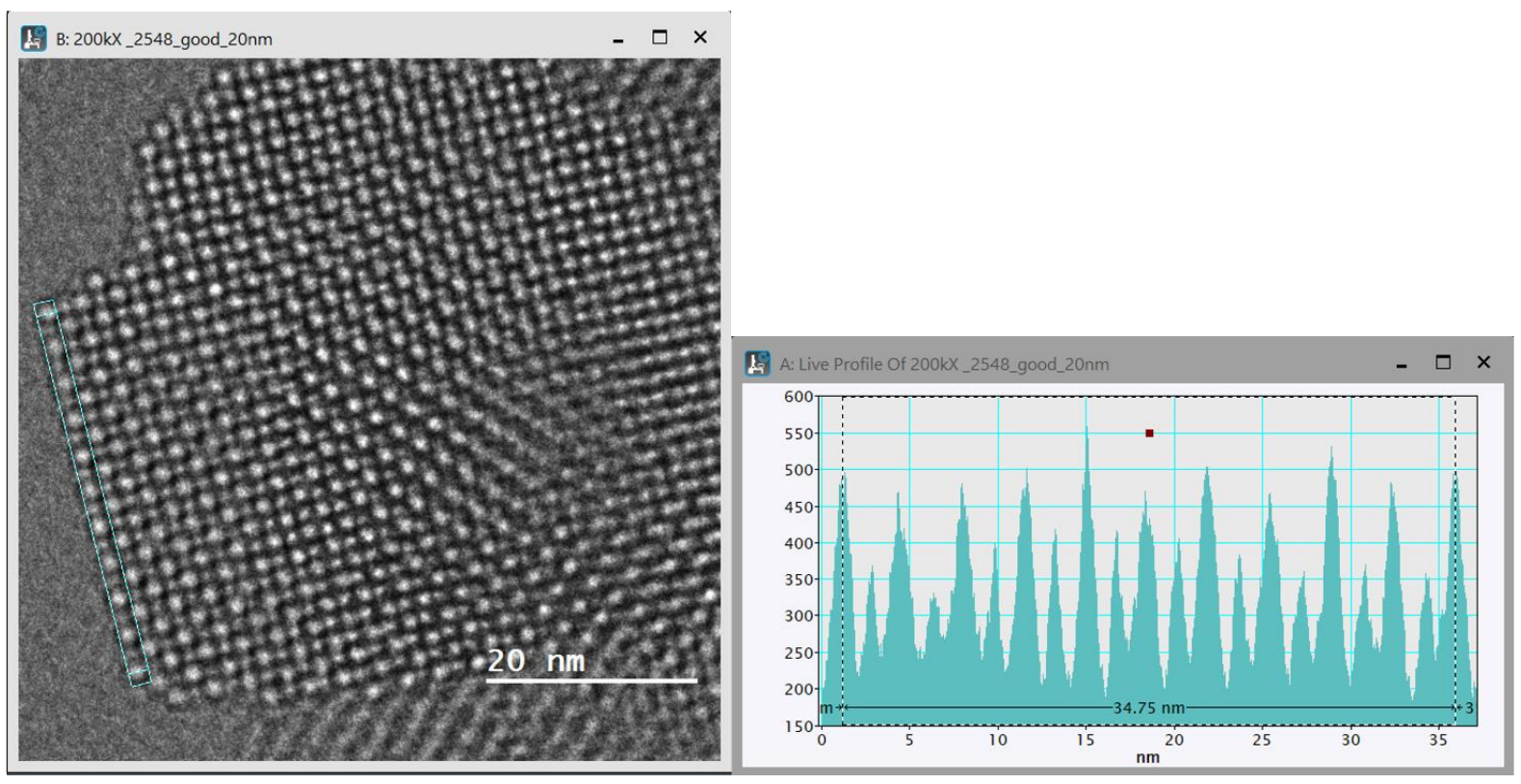

Figure S30. The distance measured between the brightest PcCo unit columns ( = 3.47 $\mathrm{nm})$ in the HR-TEM image of SPB-COF-DBA. HR-TEM image used here is the enlarged graph of Figure $3 \mathrm{~d}$ in the main text. 


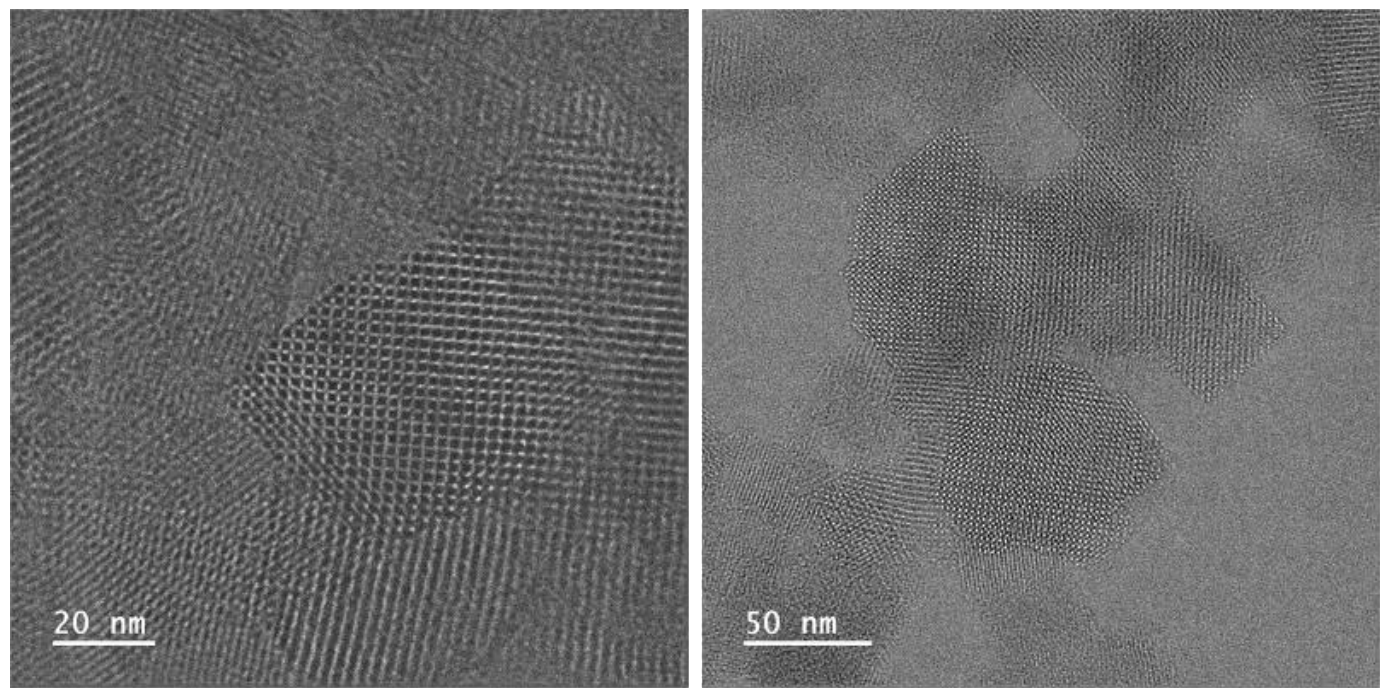

Figure S31. HR-TEM images of SPB-COF-DBA as-synthesized.

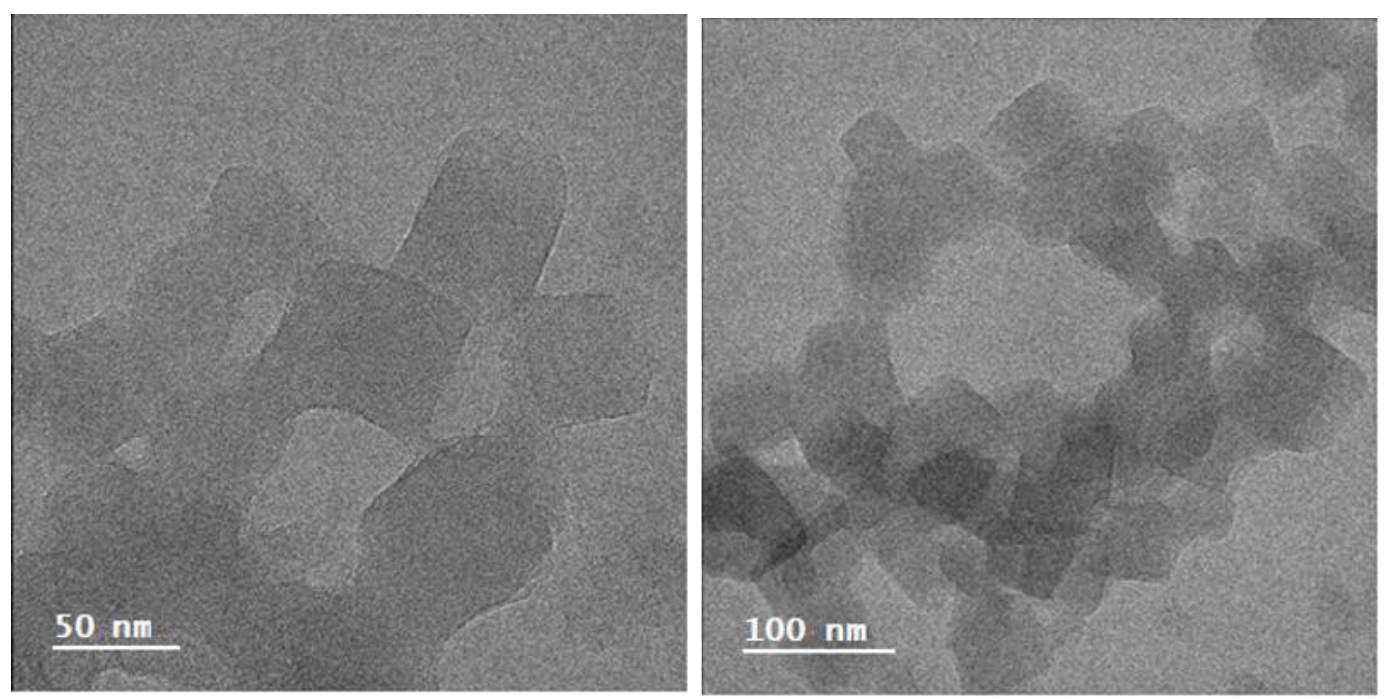

Figure S32. HR-TEM images of SPB-COF-DMA as-synthesized.
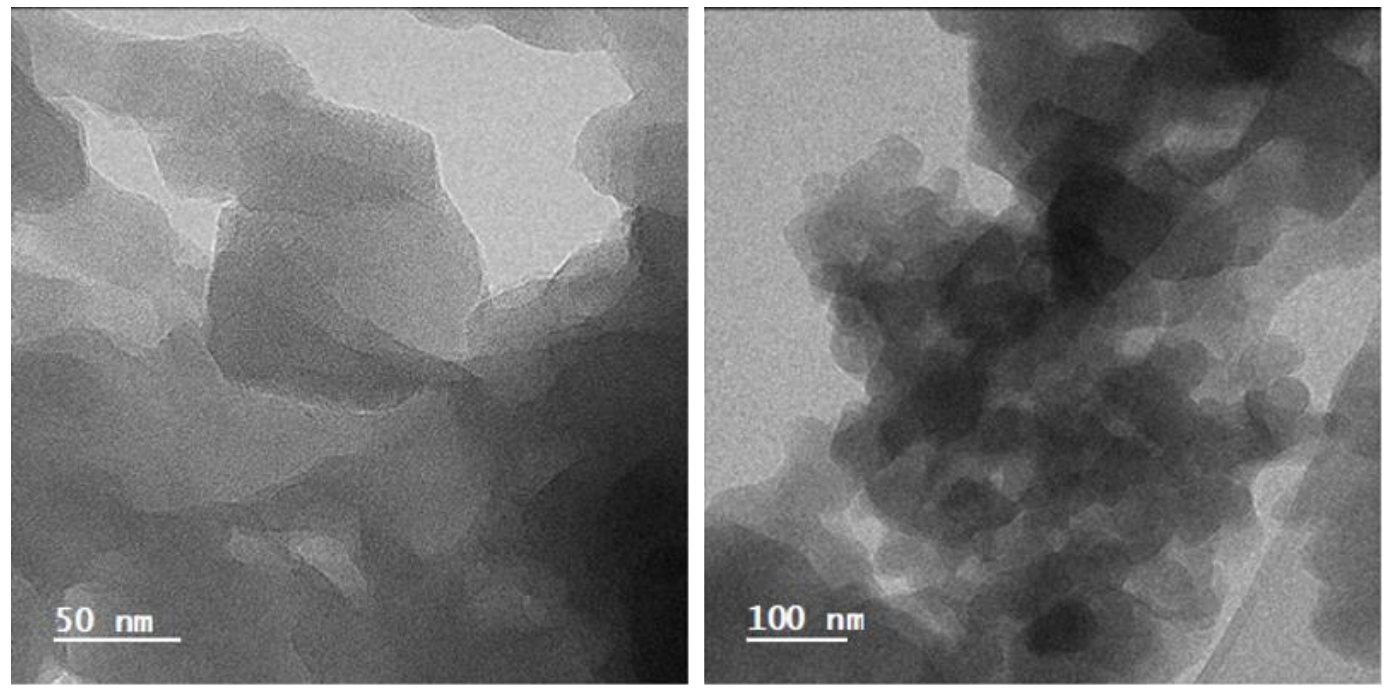

Figure S33. HR-TEM images of SPB-COF-TBA as-synthesized. 


\section{Gas sorption isotherms}
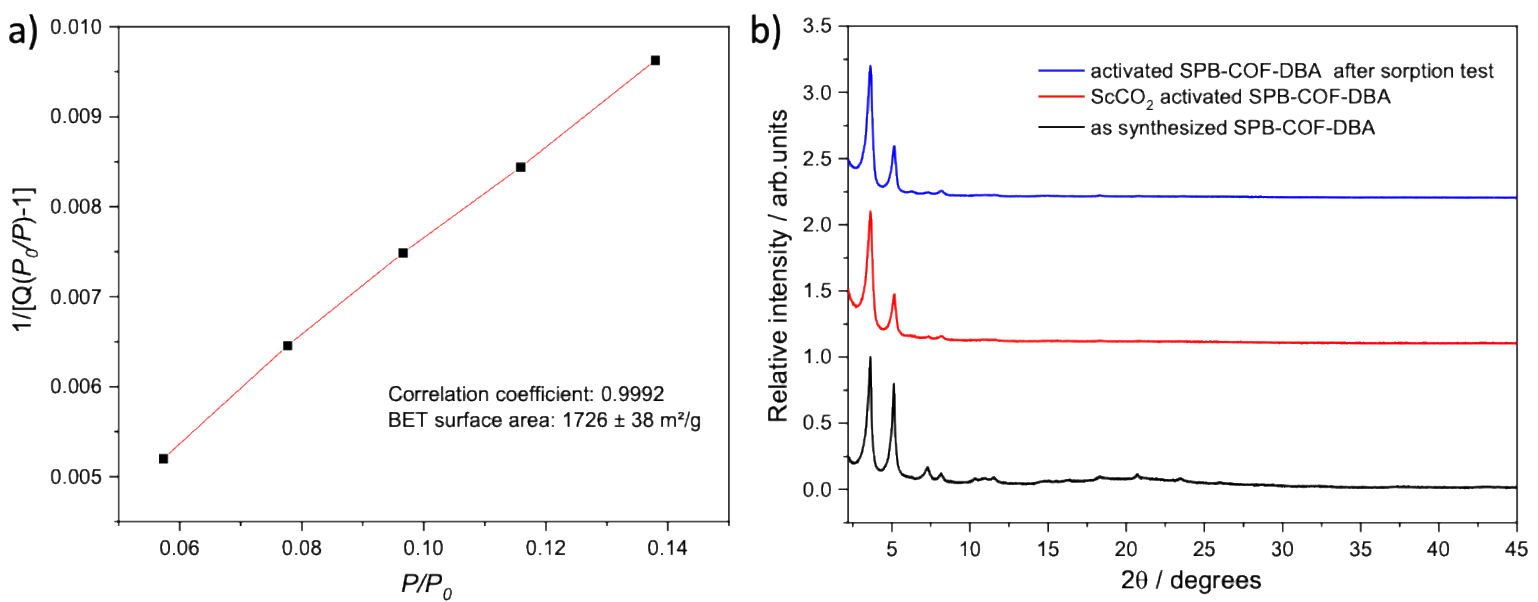

Figure S34. (a) BET surface area plot for SPB-COF-DBA. (b) PXRD comparison of SPB-COF-DBA, as synthesized, after $\mathrm{ScCO}_{2}$ activation and after sorption test. We used $\mathrm{ScCO}_{2}$ activated samples for sorption measurement.
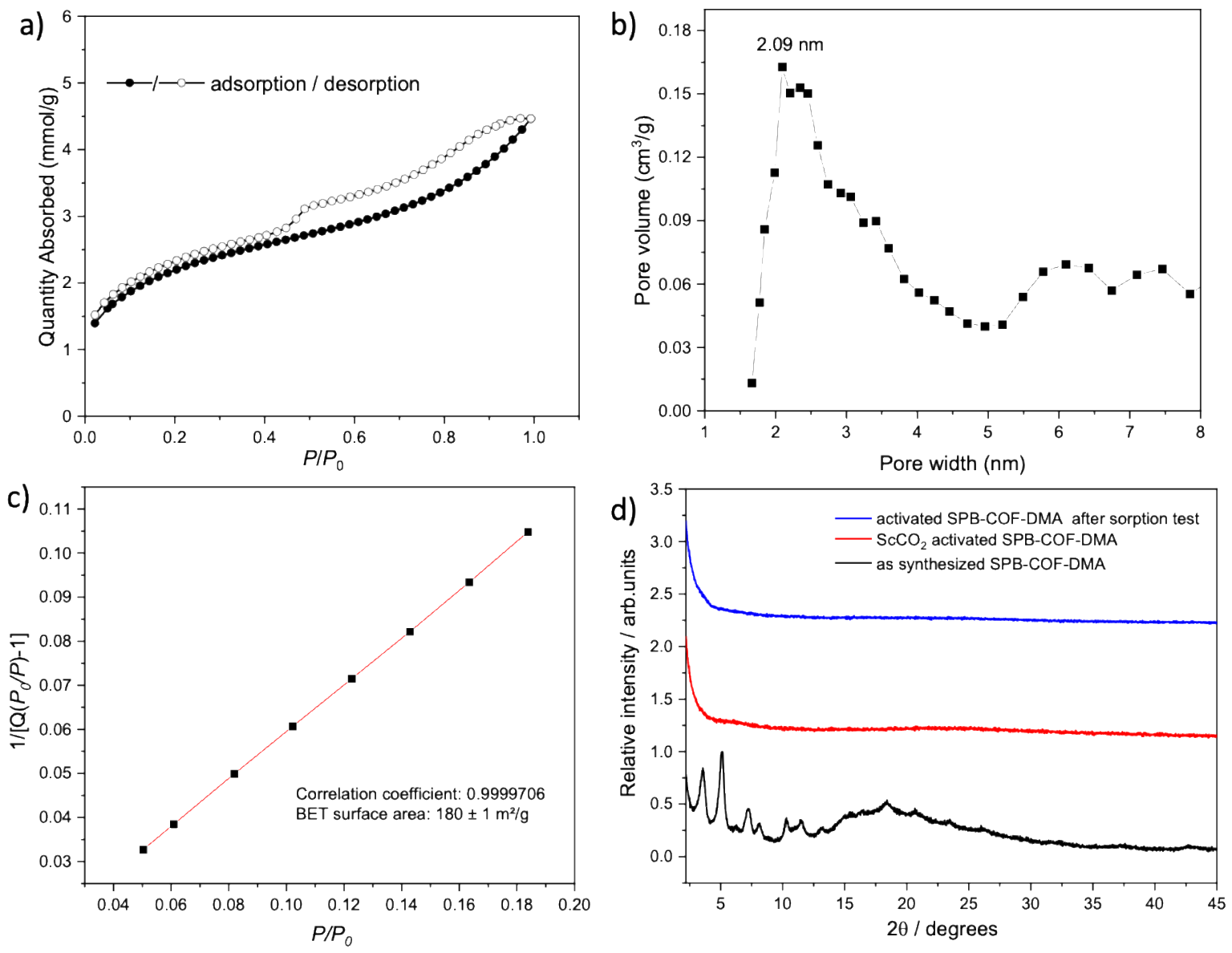

Figure S35. (a) Nitrogen adsorption/desorption isotherms for SPB-COF-DMA recorded at 77 K. (b) Pore size distribution profiles of SPB-COF-DMA calculated by NL-DFT. (c) BET surface area plot for SPB-COF-DMA. (d) PXRD comparison of SPB-COF-DMA, as synthesized, after $\mathrm{ScCO}_{2}$ activation and after sorption test. We used $\mathrm{ScCO}_{2}$ activated samples for sorption measurements. The activation of SPB-COF-DMA using $\mathrm{ScCO}_{2}$ was not successful. 

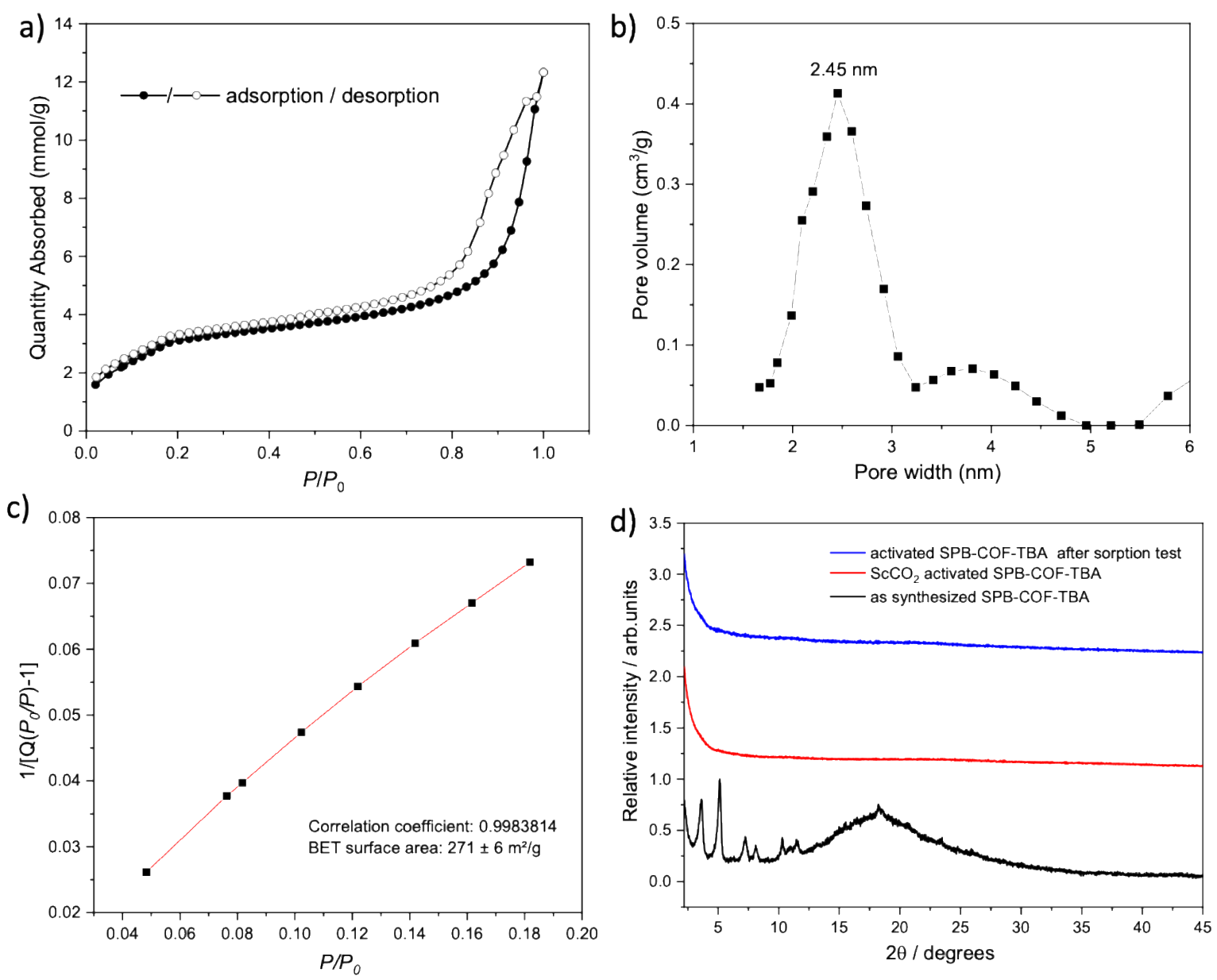

Figure S36. (a) Nitrogen adsorption/desorption isotherms for SPB-COF-TBA recorded at $77 \mathrm{~K}$. (b) Pore size distribution profiles of SPB-COF-TBA calculated by NL-DFT. (c) BET surface area plot for SPB-COF-TBA. (d) PXRD comparison of SPB-COF-TBA, as synthesized, after $\mathrm{ScCO}_{2}$ activation and after sorption test. We used $\mathrm{ScCO}_{2}$ activated samples for sorption measurement. The activation of SPB-COF-TBA using $\mathrm{ScCO}_{2}$ was not successful. 


\section{Chemical stability of SPB-COFs}

As-synthesized SPB-COF powders $(20 \mathrm{mg}$ ) were immersed in different organic solvents or aqueous solutions $(3.0 \mathrm{~mL})$ at room temperature for 24 hours. The SPB-COFs were then separated by centrifugation and washed with anhydrous acetone three times. The PXRD of these samples was then measured without further purification. The SPB-COF samples were dried in an $80^{\circ} \mathrm{C}$ vacuum oven for 6 hours before FT-IR measurement. These samples were the same batch as used for PXRD measurements. As shown below, SPBCOFs are stable in all the organic solvents tested and aqueous solutions except acidic solutions - we propose this is due to the intrinsic susceptibility of spiroborate units to hydrolyse in acidic conditions. ${ }^{11,12}$
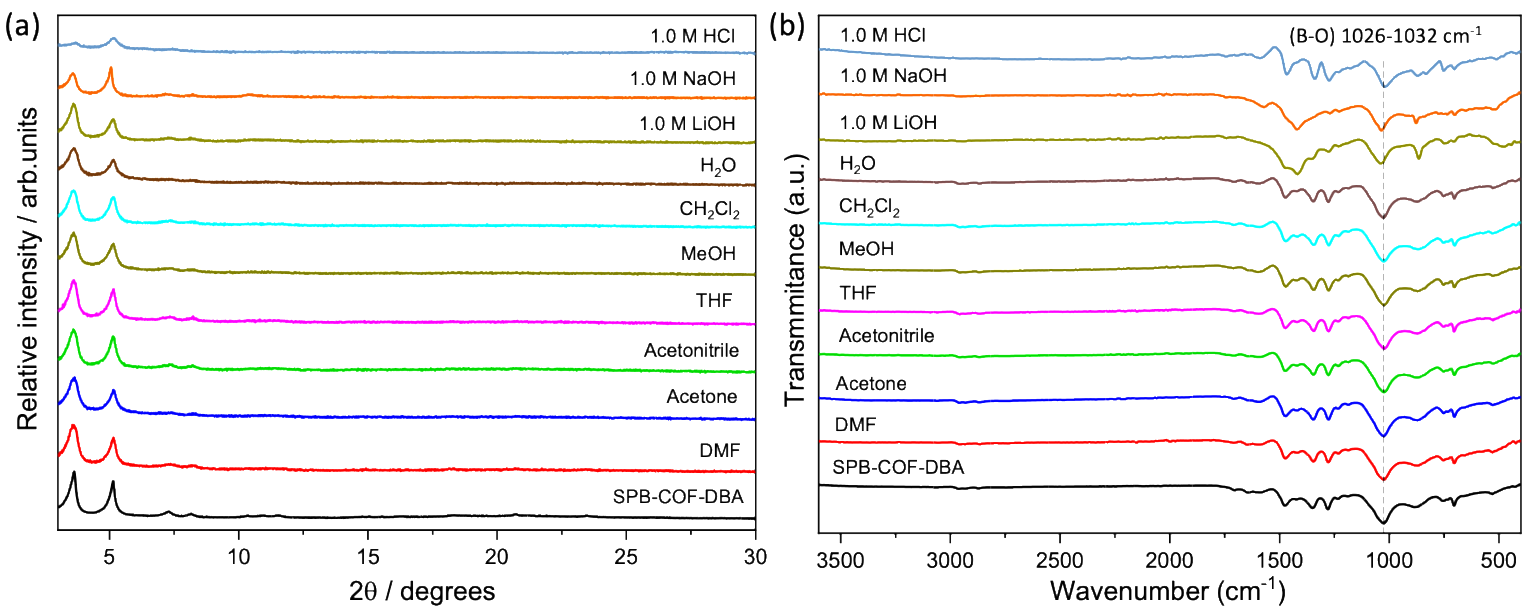

Figure S37. (a) PXRD patterns and (b) FT-IR spectra of SPB-COF-DBA before and after immersion in solvent for $24 \mathrm{~h}$.
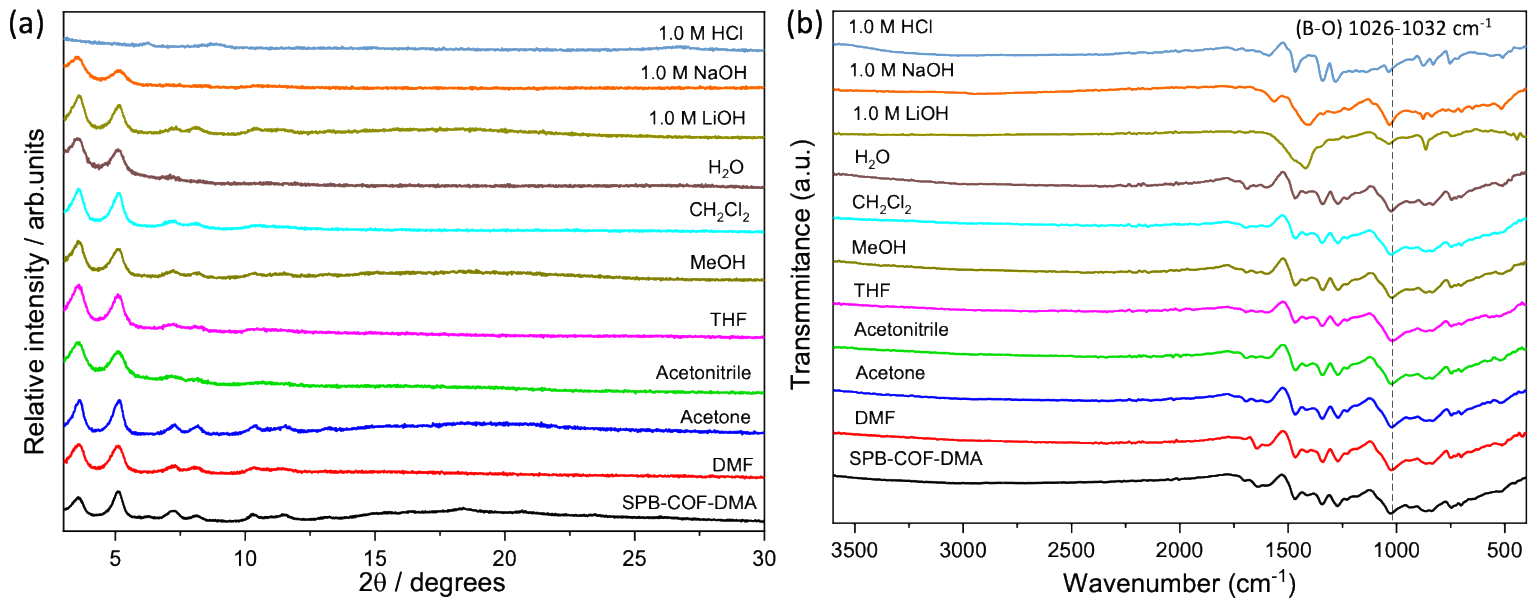

Figure S38. (a) PXRD patterns and (b) FT-IR spectra of SPB-COF-DMA before and after immersion in solvent for $24 \mathrm{~h}$. 

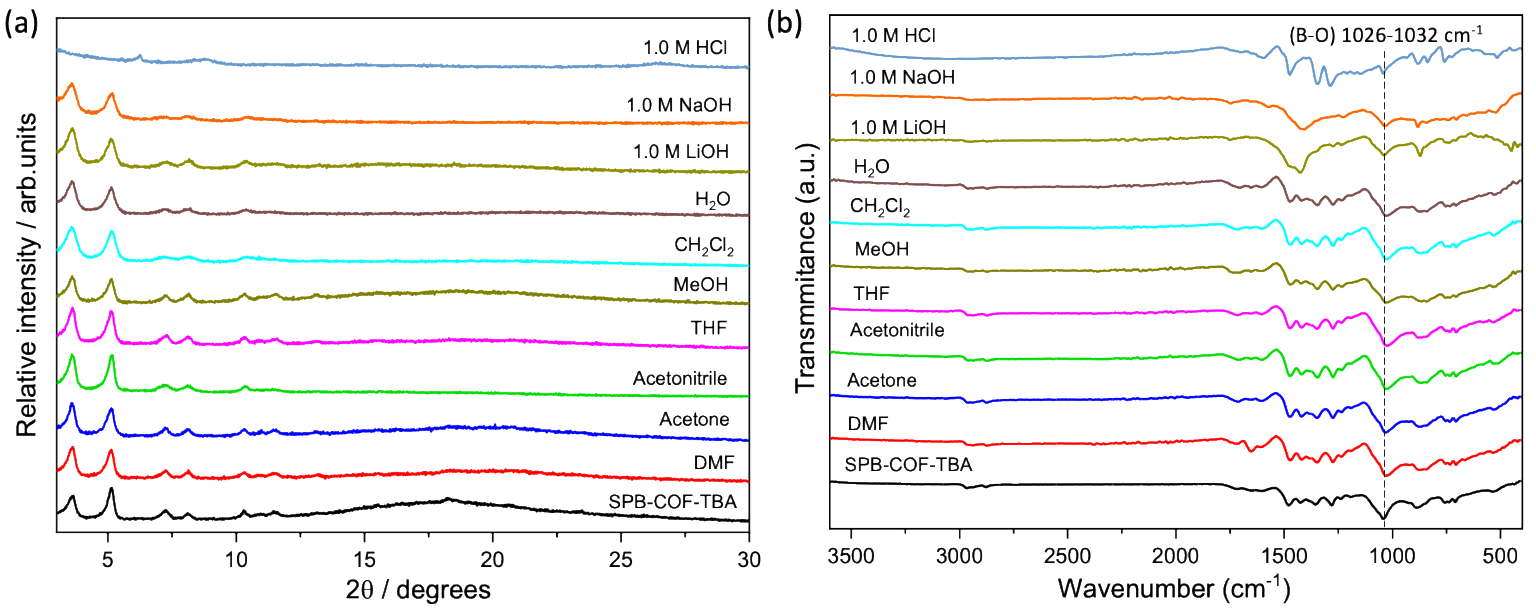

Figure S39. (a) PXRD patterns and (b) FT-IR spectra of SPB-COF-TBA before and after immersion in solvent for $24 \mathrm{~h}$.

\section{References}

(1) Sheldrick, G. M. SHELXT - Integrated Space-Group and Crystal-Structure Determination. Acta Crystallogr. Sect. A Found. Crystallogr. 2015, A71 (1), 3-8.

(2) Sheldrick, G. M. Crystal Structure Refinement with SHELXL. Acta Crystallogr. Sect. C Struct. Chem. 2015, C71 (1), 3-8.

(3) Dolomanov, O. V.; Bourhis, L. J.; Gildea, R. J.; Howard, J. A. K.; Puschmann, H. OLEX2: A Complete Structure Solution, Refinement and Analysis Program. J. Appl. Crystallogr. 2009, 42 (2), 339-341.

(4) Metz, J.; Schneider, 0.; Hanack, M. Synthesis and Properties of Substituted (Phthalocyaninato)-Iron and -Cobalt Compounds and Their Pyridine Adducts. Inorg. Chem. 1984, 23 (8), 1065-1071.

(5) Ding, X.; Feng, X.; Saeki, A.; Seki, S.; Nagai, A.; Jiang, D. Conducting Metallophthalocyanine 2D Covalent Organic Frameworks: The Role of Central Metals in Controlling $\pi$-Electronic Functions. Chem. Commun. 2012, 48 (71), 8952-8954.

(6) O’Keeffe, M.; Peskov, M. A.; Ramsden, S. J.; Yaghi, O. M. The Reticular Chemistry Structure Resource (RCSR) Database of, and Symbols for, Crystal Nets. Acc. Chem. Res. 2008, 41 (12), 1782-1789.

(7) Eddaoudi, M.; Kim, J.; Vodak, D.; Sudik, A.; Wachter, J.; O’Keeffe, M.; Yaghi, O. M. Geometric Requirements and Examples of Important Structures in the Assembly of Square Building Blocks. Proc. Natl. Acad. Sci. U. S. A. 2002, $99(8), 4900-4904$.

(8) Kresse, G.; Furthmüller, J. Efficiency of Ab-Initio Total Energy Calculations for Metals and Semiconductors Using a Plane-Wave Basis Set. Comput. Mater. Sci. 1996, 6 (1), 15-50.

(9) Willems, T. F.; Rycroft, C. H.; Kazi, M.; Meza, J. C.; Haranczyk, M. Algorithms and Tools for High-Throughput Geometry-Based Analysis of Crystalline Porous Materials. Microporous Mesoporous Mater. 2012, 149 (1), 134141.

(10) Martin, R. L.; Haranczyk, M. Construction and Characterization of Structure Models of Crystalline Porous Polymers. Cryst. Growth Des. 2014, 14 (5), 2431-2440.

(11) Ito, M.; Ikai, T.; Yamamoto, S.; Taura, D.; Ousaka, N.; Yashima, E. Chiral Guest-Induced Catalytic Deracemization of a Spiroborate-Based Double-Stranded Helicate Bearing a Bisporphyrin Unit with Acids. Chem. Lett. 2020, 49 (9), 1030-1033.

(12) Narkar, A. R.; Barker, B.; Clisch, M.; Jiang, J.; Lee, B. P. PH Responsive and Oxidation Resistant Wet Adhesive Based on Reversible Catechol-Boronate Complexation. Chem. Mater. 2016, 28 (15), 5432-5439. 